\title{
Semiconductor Nanowires: A Platform for Exploring Limits and Concepts for Nano-Enabled Solar Cells
}

\section{Citation}

Kempa, Thomas Jan, Robert Watson Day, Sun-Kyung Kim, Hong-Gyu Park, and Charles M. Lieber. 2013. Semiconductor nanowires: A platform for exploring limits and concepts for nanoenabled solar cells. Energy \& Environmental Science 6(3): 719-733.

\section{Published Version}

doi:10.1039/C3EE24182C

\section{Permanent link}

http://nrs.harvard.edu/urn-3:HUL.InstRepos:10482606

\section{Terms of Use}

This article was downloaded from Harvard University's DASH repository, and is made available under the terms and conditions applicable to Open Access Policy Articles, as set forth at http:// nrs.harvard.edu/urn-3:HUL.InstRepos:dash.current.terms-of-use\#OAP

\section{Share Your Story}

The Harvard community has made this article openly available.

Please share how this access benefits you. Submit a story.

\section{Accessibility}




\title{
Semiconductor Nanowires: A Platform for Exploring Limits and Concepts for Nano-enabled Solar Cells
}

Thomas J. Kempa ${ }^{\dagger}$, Robert W. Day ${ }^{\dagger}$, Sun-Kyung Kim ${ }^{\dagger \S}$, Hong-Gyu Park ${ }^{\S}$, Charles M. Lieber ${ }^{\dagger \ddagger}$

${ }^{\dagger}$ Department of Chemistry and Chemical Biology and ${ }^{\ddagger}$ School of Engineering and Applied

Sciences, Harvard University, Cambridge, Massachusetts 02138, USA, ${ }^{\S}$ Department of Physics, Korea University, Seoul 136-701, Republic of Korea

\begin{abstract}
:
Over the past decade extensive studies of single semiconductor nanowire and nanowire array photovoltaic devices have explored the potential of these materials as platforms for a new generation of efficient and cost-effective solar cells. This feature review discusses strategies for implementation of semiconductor nanowires in solar energy applications, including advances in complex nanowire synthesis and characterization, fundamental insights from characterization of devices, utilization and control of the unique optical properties of nanowires, and new strategies for assembly and scaling of nanowires into diverse arrays that serve as a new paradigm for advanced solar cells.
\end{abstract}




\section{Table of Contents Entry:}

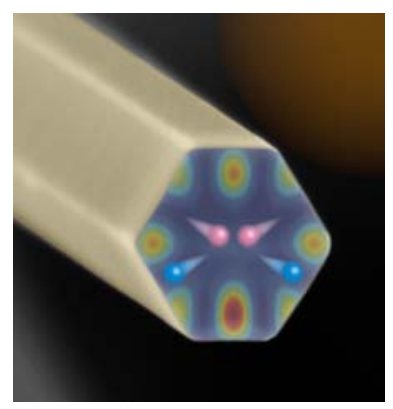

Advanced synthetic control over the electronic and optical properties of semiconductor nanowires enables testing new paradigms for advanced solar cells.

\section{Broader Context Box:}

The solar power received by the earth dwarfs global power demands by several orders-ofmagnitude. Photovoltaics convert light to electrical energy and have the potential to partially replace current energy technologies that rely on carbon-based fuels. At present, however, a lack of infrastructure and the high costs of photovoltaics prevent this. New ideas and materials are being explored to develop next-generation solar cells that could operate more efficiently and cheaply. Nanowires have emerged as one promising platform to explore such new concepts. Their small dimensions allow for efficient charge separation and light absorption properties unique as compared to bulk materials. Furthermore, the synthesis and fabrication of nanowire devices differs significantly from traditional wafer-based technologies, thus presenting new opportunities such as use of less abundant materials or cheaper substrates. Here, we discuss the benefits and remaining challenges of nanowires for PV and review progress towards understanding and optimizing the electrical and optical performance of nanowire devices. We focus on single nanowire studies that can define limits for what is achievable when multiple nanowires are assembled. Challenges and initial progress towards scaling are presented, and, for the first time, we articulate unique capabilities of solar cells derived from multiple, distinct NWs. 


\section{Author Biographies:}

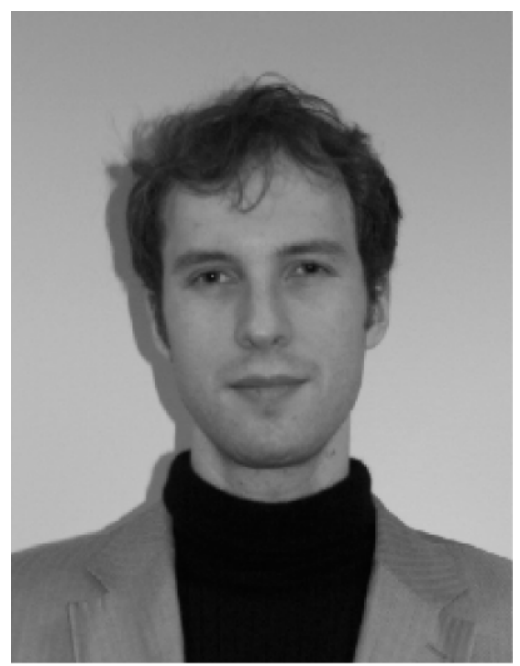

Thomas Kempa received his Ph.D. in Chemistry at Harvard University working under the supervision of Professor Charles M. Lieber. In 2004, he was the recipient of a Marshall Scholarship and spent two years at Imperial College London. His research interests include the rational synthesis of new nanoscale materials, the application of nanomaterials to challenges in energy conversion/storage and photonics, and the development of new spectroscopic tools for biology. Since 2012, he is a post-doctoral fellow in the lab of Professor Daniel G. Nocera.

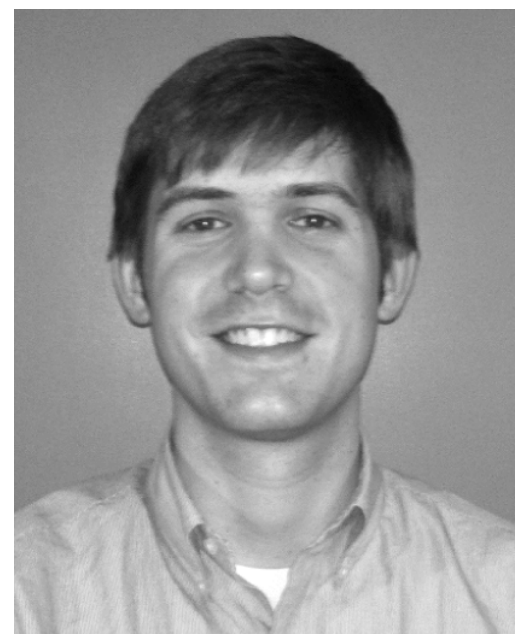

Robert Day is pursuing a PhD at Harvard University in the lab of Professor Charles M. Lieber. He obtained a BS in chemistry from the University of Richmond working under the supervision of Professor Michael C. Leopold. His current research explores the use of nanowires for photovoltaics as well as the realization of new nanowire structures through rational synthesis. 


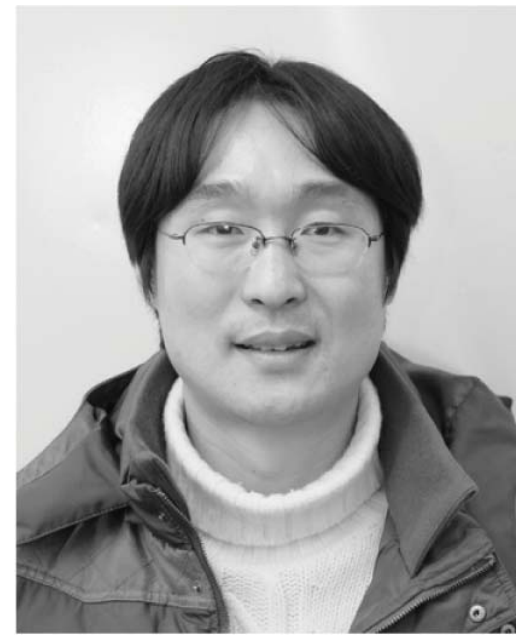

Sun-Kyung Kim has been a joint post-doctoral fellow at Harvard University and Korea University since 2010. His current research interests include Si nanowire photovoltaics and photodetectors and high-efficiency III/V light emitting diodes.

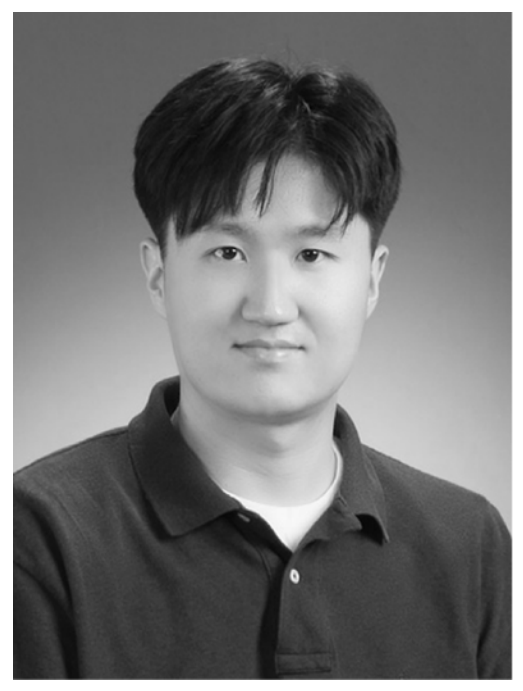

Hong-Gyu Park joined Korea University in 2007 as an Assistant Professor of Physics. His research interests include multifunctional subwavelength plasmonic devices and efficient semiconductor nanowire photovoltaics. 


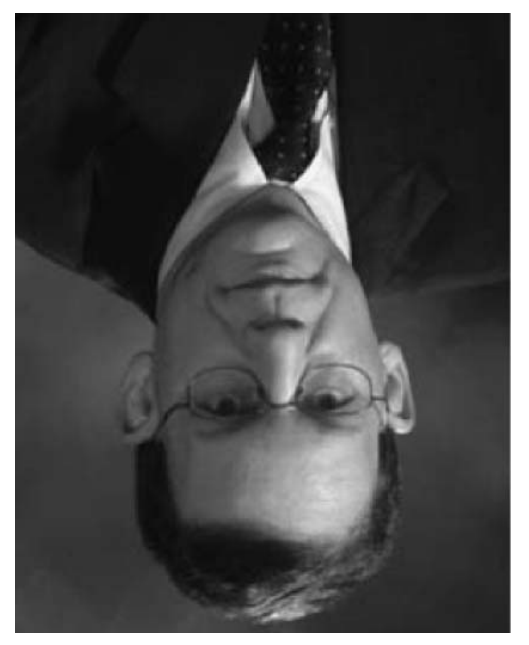

Charles M. Lieber holds a joint appointment in the Department of Chemistry and Chemical Biology, and the School of Engineering and Applied Sciences at Harvard University. His research is focused on the chemistry and physics of materials with an emphasis on the rational synthesis of new nanoscale materials and nanostructured solids, the development of methodologies for the hierarchical assembly of nanoscale materials into complex and functional systems, the investigation of fundamental electronic and optoelectronic properties of nanoscale materials, and the design and development of integrated nanoelectronics and nanoelectronic-biological systems. He is an elected member of the National Academy of Sciences. 


\section{Introduction}

The energy received by the earth from the sun in one hour could provide $80 \%$ of the energy that was consumed globally in 2008 at an average rate of $\sim 17$ TW. ${ }^{1}$ Photovoltaic (PV) solar cells are the most common form of light to electrical power converter. For over two decades, single- and poly-crystalline silicon solar cells have dominated the world market for PVs with recent average annual growth in capacity exceeding $70 \%{ }^{2}$ Nevertheless, installed PV capacity levels (40 GW in 2010) $)^{2}$ represent only $\sim 0.2 \%$ of total energy consumption, and remain low largely due to a PV efficiency to cost metric that is uncompetitive ${ }^{3}$ with conventional power generation strategies such as fossil fuel or nuclear. Although there are lower cost technologies based on thin film solar cells such as $\mathrm{CuIn}_{\mathrm{x}} \mathrm{Ga}_{1-\mathrm{x}} \mathrm{Se}_{2}$, CdTe, and amorphous silicon, ${ }^{4-7}$ these solar cells sacrifice efficiency and have not reached grid parity. ${ }^{3}$ Fundamentally, the high cost of solar power $^{3}$ is driven by expensive feedstock (e.g. high purity Si), scarcity of feedstock (e.g. rare semiconductors), and expensive material processing/purification (e.g. high temperature or high vacuum syntheses), while low efficiencies result from incomplete light absorption, carrier recombination, and thermalization losses. ${ }^{8,9}$ Seeking to break this impasse, research in nextgeneration solar cells aims to achieve greater than $20 \%$ solar to electric power conversion efficiencies at a specific cost of less than $\$ 0.50$ per peak Watt. ${ }^{3}$ While conventional technologies have steadily improved and decreased cost, there are ample opportunities to explore new materials and concepts that could lead toward high-efficiency and low-cost solar cells.

\section{Nanowires as a PV Platform}

The field of nanoscience has provided scientists and engineers with a diverse set of materials including nanoparticles, ${ }^{10-13}$ one-dimensional (1D) $)^{14-18}$ and $2 \mathrm{D}^{19,20}$ carbon materials, 
and metallic and semiconductor 1D nanowires ${ }^{21-25}$ (NWs). Over the past decade, several motivations have fueled intensive research in NWs and how these materials could increase efficiency and reduce cost in solar cells. One proposed motivation is that bottom-up syntheses could yield new device architectures difficult or impossible to realize by conventional planar fabrication strategies. For example, it has been shown that larger lattice mismatches can be accommodated during heteroepitaxial growth of nanoscale semiconductor heterostructures. ${ }^{26,27,55}$ Second, it is possible to prepare NWs with lower thermal budgets and with less complex fabrication steps than typically required for top-down manufacture of conventional solar cells. $^{28-30}$ Third, the use of flexible and/or transparent substrates could reduce cost and expand the ubiquity of solar cells in applications ranging from building-integrated to fabric-integrated PV. Fourth, the unique characteristics of light interaction with subwavelength NWs and periodic NW arrays can yield absorption comparable to conventional semiconductor films of much greater material volume. ${ }^{31}$ Finally, because of the inherently short charge separation distances in NWs with coaxial $p-n$ junctions, these structures could be used to explore new material combinations or to realize third-generation devices exploiting hot carriers, carrier multiplication, or intermediate bands, concepts which aim to overcome the Shockley-Queisser limit. ${ }^{8,9,32}$

Despite substantial progress in developing NW PVs, a number of questions remain regarding the benefits and limitations of using NWs for higher efficiency and/or more costeffective solar cells but also as compact power sources that could be integrated seamlessly with nanoelectronics. This feature review discusses strategies for implementing NWs in solar energy applications, advances in NW synthesis, insights from detailed device studies, design and control of the optical properties of NWs, and new strategies for assembly and scaling of NWs into arrays which can form the basis of solar cells. 


\section{Strategies for Implementation of Nanowire Solar Cells}

One strategy for NW solar cells relies on synthesis of NW building blocks and the subsequent assembly of these into complex multi-component devices (Figure 1). In this paradigm, sophisticated NW building blocks are synthetically encoded with distinct electrical and optical properties and then tested on the level of single devices. Results from single NW device characterization can subsequently provide feedback for the design of new functional NWs. Finally, NWs can be assembled to form highly differentiated structures that have properties distinct from monolithic or top-down processed semiconductor architectures.

To illustrate the logic of this paradigm, we propose a unique NW-enabled solar cell: the assembled NW 'multi-junction' solar cell. In an example of our concept (Figure 1C), 3 distinct NWs are synthesized to absorb efficiently blue, green, and red light. By stacking and interconnecting these horizontally-oriented NWs in the appropriate vertical order, one can realize a multi-junction solar cell with efficient absorption of solar spectrum, while simultaneously eliminating many of the significant challenges and costs associated with traditional multijunction thin-film solar cells, such as epitaxial growth or wafer-bonding of III/V materials. ${ }^{33,55}$

This bottom-up approach ${ }^{41,42}$ has been used previously to realize numerous nanoscale functional devices, ${ }^{34-40}$ and also has advantages ${ }^{43}$ for the development of next-generation solar cells: 1) synthesis can encode functionality in the form of chemical (e.g. doping) and structural (e.g. interfaces, grain boundaries, morphologies) compositions which can be tuned from the micro-, nano-, to atomic length-scales; and 2) assembly can combine diverse NW materials and structures on various substrates (e.g. glass, foil, plastic) to build a complex multi-component system with properties distinct from the individual building blocks. 
Simultaneously, numerous groups have investigated an alternative strategy based on growth of large-area NW or microwire arrays wherein the wires are oriented with their long axis perpendicular to the substrate and where the device configuration is fixed during subsequent device fabrication. ${ }^{44}$ Such an approach can deliver several benefits including: 1) device fabrication which is potentially scalable to large-area devices/modules; and 2) enhanced absorption due to long optical path-lengths and photonic effects which can persist in periodic vertically-oriented NW arrays. ${ }^{45,46}$ As an example, research groups investigating arrays of $\mathrm{Si}$ microwires have demonstrated light scattering/trapping effects from periodic and random arrangement of high-refractive index semiconductor microwire arrays. ${ }^{45}$ As another example, a ZnO NW photoanode was utilized to enhance the current density in a dye-sensitized solar cell due to increased loading of Ru-dye onto $\mathrm{ZnO} \mathrm{NW}$ surfaces and improved charge percolation. ${ }^{47}$ There are also challenges associated with this vertical NW array approach, including formation of good electrical contacts to both sides of the PV junction and uncertain cost competitiveness relative to traditional technologies stemming primarily from reliance on preparation of NW arrays from crystalline wafers. Detailed discussion of vertically-oriented arrays is beyond the scope of this review but has been recently reviewed. ${ }^{31,93}$

\section{Nanowire Synthesis, Characterization and Control}

Nanowire PV devices can incorporate $p$-type/intrinsic/n-type (p-i-n) dopant modulation for carrier separation in 2 distinct motifs: (1) axial geometry with junction encoded along the growth direction of the NW, and (2) radial geometry with junction encoded between the core and coaxial shells. Schematics of a single $p-i-n$ axial and radial nanowire diode are shown in Figure 2, where pink, yellow, and blue regions denote $p$-type, intrinsic, and $n$-type diode segments, 
respectively. In these structures, electron-hole pairs are generated upon absorption of photons whose energies are equal to or greater than the band-gap of silicon $\left(E_{\mathrm{g}}=1.12 \mathrm{eV}\right.$ for singlecrystal silicon). Carrier generation and separation are most efficient within the depletion region due to the built-in field established across the $p$-i-n junction. ${ }^{34-36,48}$ Photogenerated holes (electrons) drift in the presence of the electric field through the $p$-type (n-type) regions and are ultimately collected as photocurrent by ohmic metal contacts. In the axial configuration, the $p$ type and $n$-type regions can be made arbitrarily short since their main purpose is to provide contact to the junction encoded within the nanowire. One attractive consequence of this is that the active device area can be kept small so as to enhance integration with other nanodevices or nanoelectronic circuits. In the radial configuration, the $p$-i-n interface extends along the length of the nanowire allowing carrier separation to take place in the radial versus the longer axial direction. In this structure, carrier collection distances are smaller or comparable to the minority carrier diffusion length ${ }^{4,46}$ meaning that photogenerated carriers can reach the $p$-i-n junction with high efficiency without substantial bulk recombination, even in poor-quality or low-mobility materials.

\subsection{Synthesis of Nanowires}

Semiconductor NWs are typically synthesized using the now well-established metal nanocluster-catalyzed vapour-liquid-solid (VLS) growth process. ${ }^{21,22,41,49}$ In this process (Figure 3A), a vapour-phase semiconductor source dissolves in the liquid metal/semiconductor droplet maintained at a temperature near or above the metal/semiconductor eutectic point. Continued supply of the gaseous source supersaturates the droplet and the chemical potential between the gas-liquid and liquid-solid interfaces acts as a driving force for nucleation of the solid 
semiconductor. During growth, the liquid-solid interface is the site for continued incorporation of crystalline solid material into the elongating NW.

Axial NWs are obtained through VLS growth as illustrated in Figure 3C and Figure 3E. Growth of NW axial heterostructures entails use of various reactant sources (e.g. dopant or other semiconductor precursors) and it is therefore important to select a nanocluster catalyst that can maintain a stable eutectic under compatible conditions. Radial or core/shell heterostructures are obtained by shifting growth conditions from VLS to a vapour-solid growth regime where dissociation and deposition of reactants is promoted on the NW surface (Figure 3B) resulting in concentric shells. ${ }^{27}$ Radial NWs encode function in the form of coaxial shells (Figure 3D, F) and afford several advantages as compared to VLS-grown axial NWs, including: (1) orthogonalization of carrier separation and collection into radial and axial components, (2) atomically sharp interfaces are accessible due to the layer-by-layer vapour-phase growth mechanism, and (3) greater freedom to synthesize new structures because of the broad range of parameters for shell growth, which will be discussed below.

A diversity of solution-phase and gas-phase techniques exists for the synthesis of semiconductor NWs. Chemical vapor deposition (CVD) has been broadly adopted for growth of group IV, III/V, and II/VI semiconductor NWs and, in a few instances, molecular beam epitaxy (MBE) from gas ${ }^{50}$ and solid-state precurors has also been utilized. Metal-organic chemical vapour deposition (MOCVD) ${ }^{51}$ has been extensively applied for growth of compound semiconductor NWs. ${ }^{54-56}$ Silicon core/multi-shell NWs with high-quality electronic interfaces were recently synthesized using a low-pressure CVD method tailored to elicit a polymorphic crystal structure with very low defect densities. ${ }^{34}$ A scanning electron microscopy (SEM) image 
of such a Si NW (Figure 3G) reveals smooth facet surfaces distinct from the disordered and unfaceted nanocrystalline morphologies previously reported. ${ }^{36}$ In this work, the high-quality NW material led to the first demonstration of Si NW solar cells with large $V_{\text {oc's of }} 0.5 \mathrm{~V}$. In addition, low-temperature, solution-phase doping of CdS NWs with $\mathrm{Cu}$ (Figure 3H) has been used to form active devices with good electrical properties, including $V_{\mathrm{OC}}$ 's of $=0.6 \mathrm{~V}$ and efficiencies of $5.4 \%{ }^{52}$ In addition, the Samuelson group has pioneered the use of molecular organic vapour phase epitaxy (MOVPE) to grow III/V NWs. In a recent work, the Samuelson group demonstrated the ability to selectively incorporate polytypic and twin-plane superlattices in InAs NWs (Figure 3I) and showed how these crystallographic manipulations can be tuned with welldefined synthetic parameters including temperature and diameter. ${ }^{53}$ Together, these reports underscore the range of synthetic systems available for growth of semiconductor NWs.

\subsection{Nanowires: Diversity in Material, Structure, and Morphology}

Synthetic advances in the growth of group IV, III/V, and II/VI semiconductor NWs have correspondingly enabled substantial progress in transistor, laser, and solar-cell devices. For example, Ge/Si core/shell nanowires were first prepared by nanocluster-catalyzed VLS-growth of crystalline Ge cores followed by low-temperature deposition of an a-Si shell that was subsequently annealed. ${ }^{27}$ The resulting high-resolution transmission electron microscopy (TEM) images and energy dispersive x-ray spectroscopy (EDS) maps of Si and Ge (Figure 4A) confirmed that the nanowire heterostructures possessed abrupt and crystalline interfaces. MOCVD synthesis of defect-free InGaN/GaN NWs with 26 multiple quantum well (MQW) structures $^{54}$ (Figure 4B, C) further demonstrated the potential for implementing complex structures at the nanoscale using materials whose large lattice mismatches could not be easily 
accommodated in thin films. ${ }^{57}$ Finally, it has been demonstrated recently that axial NWs can be induced to kink at specific points through transient modulation of growth pressure and that multiple kinks can be encoded through iterative application of such modulations. ${ }^{58}$ In addition to Si and Ge kinked NWs, group II/VI multiply kinked CdS NWs were synthesized (Figure 4D), thus demonstrating the generality of this approach.

In addition to the many semiconductor materials and structural types accessible via controlled synthesis, unique morphologies are possible through tailored synthesis of Si NWs. It is possible to synthesize NWs with hexagonal, octagonal, and rectangular cross-sections (Figure 5A) through modification of the aforementioned core/shell synthesis (e.g. shell growth temperature, silane partial pressure, etc.). Specifically, bright-field TEM images of Si core/multishell NWs sectioned ca. perpendicular to their axes reveal a hexagonal cross-section ${ }^{34}$ with welldefined surface facets (Figure 5B). By growing the final, n-type doped, shell of this NW at $860^{\circ} \mathrm{C}$ instead of the typical $775^{\circ} \mathrm{C}$, Si deposition can be accelerated on the $\{113\}$ facets ${ }^{59}$ leading to a NW with an approximately rectangular cross-section (Figure 5B). Control of morphology is also possible through subtle modulations in crystal structure within the crosssection of a NW. A dark-field TEM image (Figure 5C) of the hexagonal Si cross-section shown in Figure 5B reveals more detail regarding the internal crystal structure of the NW. A thin polycrystalline region surrounding the $100 \mathrm{~nm}$ NW core is visible as brighter contrast, as are other distinct features such as grain boundaries. Although structural heterogeneity exists in the NW, its electron diffraction pattern (Figure 5C) is consistent with reflections from the diamond cubic lattice of $\mathrm{Si}$ oriented along $<211>$, from which we conclude that the individual crystal domains share the same orientation as the core. Interestingly, variations in internal crystal morphology and cross-sectional morphology can have significant effects on light absorption in 
these subwavelength cavities. These features and their implications for NW PV devices will be discussed in detail below.

The realization of well-defined nanoscale interfaces for charge separation is vital to the electrical performance of NW solar cells. Representative high-resolution TEM images of crosssections of core/multi-shell Si NWs (Figure 6A) show clear (111) lattice fringes continuing up to the amorphous $\mathrm{SiO}_{2}$ coating at the outermost edge of the NW. ${ }^{34}$ These data reveal that over an area of the cross-section spanning the width of the $p-i-n$ junction there are minimal crystal defects. Low defect densities are an important criterion for high-quality electrical interfaces in photodetector, photonic, or solar cell applications. In addition, EDS mapping data were used to characterize the phosphorous dopant and oxygen profiles in a Si core/shell cross-sectional sample (Figure 6B). The phosphorous profile exhibits two peaks coincident with the left and right sides of the outer n-shell which have peak widths, 20-30 nm, in good agreement with the

expected n-shell thickness of $25 \mathrm{~nm}$ based on measured growth rates. ${ }^{34}$ Such measurements verify that well-defined, nanoscale dopant variations can be encoded in core/shell NWs and, more generally, demonstrate the control possible with bottom-up synthesis.

\section{Device Electrical Transport and Performance}

Fundamental studies of NWs for next-generation solar cells involves two important steps: (1) synthesis of NW components with precisely defined structural and chemical properties, and (2) elucidation of PV properties at the single NW level, which permits determination of the optoelectronic properties, intrinsic limits, and potential benefits of NWs for solar cells.

For device fabrication, NWs must be transferred from their growth substrates to a host substrate that will support the NW in its final device configuration. Core-shell Si NWs are 
typically transferred to $\mathrm{Si}_{3} \mathrm{~N}_{4}$ substrates using dry transfer ${ }^{34}$ or contact printing techniques. ${ }^{60,61}$ Subsequently, a portion of a single NW is protected with a lithographically defined etch mask and then immersed in etchant so that the exposed portion can be removed to the p-type core. ${ }^{34}$ Once metallic contacts are selectively defined to n- and p-type portions of the core/shell device, the single NW device is complete (Figure 7A). Axial devices ${ }^{35}$ follow a very similar fabrication process, but without the need for etch masks. NWs containing other semiconductors may require different substrates, etch masks, etchants, etching processes, or metal contacts from those developed successfully for Si NWs. ${ }^{34-36}$

Investigation of the $I-\mathrm{V}$ transport characteristics (Figure 7B) of single core/multi-shell NW devices ${ }^{34}$ and core/nanocrystalline-shell NW devices ${ }^{36}$ revealed several important trends. First, the higher $V_{\mathrm{OC}}$ value, $0.48 \mathrm{~V}$, was obtained from the optimized core/multi-shell geometry and represents a substantial, two-fold improvement over core/nanocrystalline-shell ${ }^{36} \mathrm{NWs}$, and is the highest $V_{\text {OC }}$ reported to-date for a single Si NW device. Significantly, one can attain $V_{\mathrm{OC}}$ 's $>$ $0.47 \mathrm{~V}$ from devices with diameters as small as $200 \mathrm{~nm}$, demonstrating that much larger micronscale diameters ${ }^{62}$ are not necessary to achieve a good $V_{\text {OC. }}$ Second, the core/multi-shell device exhibits a larger fill factor (FF) of 73\%, which is substantially higher than the $55 \%$ FF reported previously. ${ }^{36}$ Third, while the core/nanocrystalline-shell geometry attains the higher $J_{\text {SC }}$ of 23.8 $\mathrm{mA} / \mathrm{cm}^{2}$, core/multi-shell NW devices exhibit $J_{\mathrm{SC}}$ values, $8-10 \mathrm{~mA} / \mathrm{cm}^{2}$, substantially larger than expected for an equivalent thickness of bulk Si. ${ }^{63}$ The physical origin of this striking observation will be discussed in Section 6 .

Recently, finite element method (FEM) simulations ${ }^{96}$ showed that both axial and radial NW PVs are capable of achieving high $V_{\text {OC }}$ values of $\sim 0.7$ V. For axial NWs, simulations show that surface recombination near the exposed junction is primarily responsible for limiting $V_{\mathrm{OC}}$ 
below 0.7 V. For radial NWs, bulk minority carrier lifetimes $<10$ ns are primarily responsible

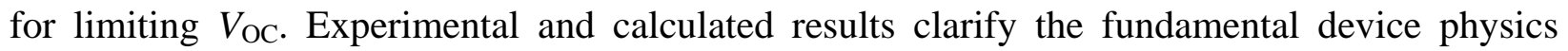
limits that must be surmounted to achieve high $V_{\text {OC }}$ NW PVs.

To assess the influence of shell architecture on electronic properties of core/multi-shell NWs, we examined the junction leakage current for four distinct types of structures. A plot of $V_{\mathrm{OC}}$ versus the logarithm of the ratio of short-circuit current $\left(I_{\mathrm{SC}}\right)$ to dark saturation current $\left(I_{0}\right)$ for sixteen total devices (Figure 7C) is linear, as expected, with a monotonic increase in $V_{\mathrm{OC}}$ with decreasing $I_{0 .}{ }^{63}$ Representing a 1000-fold improvement over previous nanocrystalline-shell NW devices, ${ }^{36}$ the $p /$ in device with $V_{\mathrm{OC}}=0.48 \mathrm{~V}$ has a dark saturation current $I_{0}=1.1 \mathrm{fA}$ and dark saturation current density $J_{0}=0.34 \mathrm{fA} / \mu \mathrm{m}^{2}$. This dramatic reduction in leakage current directly correlates with the two-fold improvement in $V_{\mathrm{OC}}$. Furthermore, the steady reduction in $I_{0}$ (increase of $V_{\mathrm{OC}}$ ) from the $p / n$ to $p / p n$ to $p / i n$ device underscores how electrical performance is a sensitive function of synthetically encoded junction properties. Notably, the temperature coefficient of $V_{\mathrm{OC}}$ extracted from $I / V$ data acquired down to $60 \mathrm{~K}$ is $-1.9 \mathrm{mV} / \mathrm{K}$ and close to the value $\mathrm{d} V_{\mathrm{OC}} / \mathrm{dT}=-1.7 \mathrm{mV} / \mathrm{K}$ calculated for bulk single-crystalline $\mathrm{Si}$ solar cells, ${ }^{64}$ which attests to the good transport characteristics of core/multi-shell NW devices. Interestingly, data obtained on core/multi-shell NW devices with lengths spanning over 1 order of magnitude (Figure 7D) exhibit linear scaling of $I_{\mathrm{SC}}$ and nearly constant $J_{\mathrm{SC}}$. This result confirms that $\mathrm{NW}$ device performance is preserved over a large range of NW lengths and encourages use of these building blocks in future large-area arrays which could be fabricated solely by standard photolithography. Together, these data demonstrate that precise synthetic control of NW structures is a powerful determinant of improvements in $V_{\mathrm{OC}}, \mathrm{FF}$, and $I_{0}$. 
Several groups have proposed that metal nanocluster catalysts, especially gold, used in NW growth could enhance recombination and adversely affect $V_{\mathrm{OC}}$ in NW devices. ${ }^{65}$ To further investigate this possibility, NWs were synthesized using $\mathrm{Al}$ catalyst ${ }^{65}$ which, unlike $\mathrm{Au}$, does not act as a mid-band gap trap state in $\mathrm{Si}^{66}$ Nevertheless, the best illuminated $I$-V curve for the core/multi-shell diode geometry (Figure $8 \mathrm{~A}$, grey curve) yields a $V_{\mathrm{OC}}, 0.23 \mathrm{~V}, \sim 2$ times smaller than the value for analogous Au-catalyzed devices. Furthermore, even devices where Au was removed by wet-chemical etching of as-grown NW cores (Figure 8, red curve) yielded a $V_{\mathrm{OC}}$, $0.44 \mathrm{~V}$, similar to the best results obtained on core/multi-shell devices prepared without removal of the catalyst. Together, these results suggest that proposed Au impurities do not significantly lower $V_{\mathrm{OC}}$ and again affirm that the overall quality of the core/multi-shell structure, as dictated by synthesis, is the most important driver of good electrical performance.

Several reports have also focused on the role of NW surface states in recombination of charge carriers. Using near-field scanning optical microscopy (NSOM) to locally excite charge carriers in a reverse-biased axial NW device, researchers found that a $10 \mathrm{~nm}$ a-Si shell can serve as effective passivation with a 100 -fold reduction in surface recombination. ${ }^{67}$ Furthermore, Kelzenberg et al. have performed scanning photocurrent mapping experiments on single $\mathrm{Si}$ microwire devices with $p$ - $n$ junctions and diameters between 1.2 and $1.8 \mu \mathrm{m}$. They discovered that while a thin a-Si:H passivation layer can provide a surface recombination velocity of $S \sim 450$ $\mathrm{cm} / \mathrm{s}$, a-SiN $\mathrm{x}: \mathrm{H}$ provides superior passivation with $S<<70 \mathrm{~cm} / \mathrm{s}$ and a correspondingly very long minority-carrier diffusion length $L_{\mathrm{n}}>>30 \mu \mathrm{m} .{ }^{62}$ Together, these results argue that with proper passivation it is possible to improve the electrical performance of NW devices. 


\section{Light Absorption in Nanowires}

In addition to good electrical performance, a solar cell must absorb significant light in order to achieve high efficiency. Conventional semiconductor devices, such as bulk crystalline or thin-film solar cells, achieve greater light absorption by use of thicker absorbing layers ${ }^{68}$ and/or anti-reflection techniques (e.g. surface texturing ${ }^{69-71}$ or a quarter-wave dielectric coating ${ }^{72,73}$ ). However, increased material usage and additional fabrication steps typically increase cost. $^{74}$ Absorption of light by NWs is significantly distinct from bulk material absorption. Several groups have shown that subwavelength size, high-refractive index NWs can absorb a significant amount of light using a fraction of the volume needed with bulk semiconductor material. ${ }^{34,59,75}$

To develop more efficient NW PV devices, significant effort has focused on how the

absorption properties of NWs depend on material, size, and cross-sectional morphology. ${ }^{34,59,75-77}$ Synthetic control over these parameters may allow the design of NW devices with absorption characteristics distinct from traditional crystalline wafer or thin-film structures. ${ }^{34,59}$ In addition to light absorption at the single NW level, horizontally ${ }^{75,78}$ - or vertically-oriented ${ }^{45,79} \mathrm{NW}$ arrays with appropriately chosen pitch size have been shown to exhibit broadband anti-reflection as well as enhanced light-scattering. The following sections discuss the origin of distinct light absorption, and strategies to tune light absorption in single NWs and their assembled structures. We emphasize that synthesis affords a high degree of control to tune and optimize light absorption in these materials for PV.

\subsection{Introduction and Basic Theory}


Semiconductor NWs are subwavelength optical cavities that can support resonant modes. To exploit the optical properties of NWs it is necessary to quantify their optical resonances through calculation ${ }^{34,59,75-77,80,81}$ and measurement ${ }^{34,59,75-77,82}$. Simulations illustrate how a NW interacts with incident light and can describe in detail the properties (e.g. profile and amplitude) of resonant modes. Measurements, typically photocurrent spectra of single NW devices, provide crucial verification of simulation and combined with calculations allow for design of new NWbased optical cavities.

Analytical Lorentz-Mie theory ${ }^{75,76,80,81}$ or numerical full-field electromagnetic simulations ${ }^{34,59,77}$ have been widely used for studying light-matter interaction in nanowires. For example, in finite-difference time-domain (FDTD) simulations, a normally incident plane wave with a specific wavelength and polarization state (i.e. transverse-electric (TE) or transversemagnetic (TM)) interacts with a NW cavity, as shown in Figure 9A. The calculated electric field distribution illustrates that a Si NW can, at specific wavelengths, absorb incident photons beyond its physical cross-section; this phenomenon is called the 'optical antenna effect'. $34,75,77,83,84$ The absorption mode profile at a specific wavelength (Figure 9B) can be obtained by recording $J \cdot E$ at each grid point within a NW, where $J$ and $\mathrm{E}$ are the polarization current density and electric field, respectively. The NW cavity modes have localized spatial profiles, including Fabry-Perot, whispering gallery, and higher-order complex modes at particular wavelengths. A combination of optical antenna effects and distinct resonant modes in NWs leads to light absorption that differs significantly from bulk material absorption.

Experiments to verify and quantify simulated optical properties of NWs have been carried out by measuring photocurrent spectra of single NW PV devices ${ }^{34,59,62}$ or photodiodes $^{75-77,85}$. For example, external quantum efficiency (EQE) values of $\sim 0.15$ have been 
reported for micro-wire devices based on Al-Si Schottky junctions. ${ }^{85}$ Relative EQE values have been also reported for $\mathrm{Si}^{75,77}$ and $\mathrm{Ge}^{76}$ nanowire devices acting as photodetectors. Significantly, measurement of the absolute EQE from Si NW PV devices has been reported in several instances. ${ }^{34,59}$ Shown in Figure 9C is a representative result of the absolute EQE versus wavelength acquired for a $p-i-n$ core/multi-shell NW device and a simulated spectrum of the same structure. Both the experimental and simulated spectra are highly structured, with good agreement in the peak wavelengths and amplitudes, where the only adjustable parameter in the FDTD simulation was the size of the NW. By comparing the experimental and simulated spectra, peaks in the experimental spectrum are assigned to specific resonant absorption modes (Figure 9B). Finally, the EQE spectrum approaches and even exceeds unity for wavelengths in the range of 400-500 nm which is a manifestation of the optical antenna effect. ${ }^{34,62,83}$ This effect is purely classical and caused by the sub-wavelength diameter of the NW. To calculate EQE from photocurrent or from simulated absorption data, the projected area of the NW (normal to substrate plane) was used. In the end, EQE values greater than unity can be observed because the absorption cross-section of a single NW can exceed its physical cross-section for some photon energies. Figure 9D presents the normalized absorption spectra for Ge NW photodetectors with diameters ranging from 10 to $110 \mathrm{~nm} \cdot{ }^{92}$ Taken together, these findings demonstrate that semiconductor NWs can sustain distinct wavelength-dependent absorption characteristics across a large range of the solar spectrum.

\subsection{Size and Morphology Effects}


One attractive feature of subwavelength size cavities is that fine changes to their structure can lead to dramatic alteration of their optical properties. ${ }^{86-88}$ Recent studies have revealed how morphological changes (i.e. size and cross-sectional morphology) in NWs influence their absorption characteristics. ${ }^{34,59,75-77}$ In general, the ability to significantly tailor absorption in NWs as compared to conventional planar structures could drive development of efficient photovoltaic devices as well as other photonic applications.

The absorption behavior of $p-i-n$ NWs with hexagonal cross-sections was investigated as a function of diameter. EQE spectra for the core-shell Si devices with diameters of 170, 280, and $380 \mathrm{~nm}$ (Figure 10A, black straight line) and simulated spectra from the same structures (Figure 10A, red dashed line) highlight several features. First, the number of measured peaks increases with increasing NW diameter. Second, identical absorption modes shift to longer wavelengths with increasing NW size. For instance, the peaks at 445, 620, and $795 \mathrm{~nm}$ for the small, intermediate and large sized devices, respectively, correspond to the same Fabry-Perot type modes. For PV purposes, enhanced current density $\left(J_{\mathrm{SC}}\right)$ can be achieved by tuning the absorption peaks so that they are better matched in wavelength to the maximum irradiance of the solar spectrum..$^{34,59}$

The $J_{\mathrm{SC}}$ of a NW is calculated by integrating the product of its EQE spectrum with the solar spectrum. A plot of the total photocurrent per unit area (Figure 10B, dashed red) and per unit volume (Figure 10B, dashed black) as a function of NW diameter is obtained from simulation. From this data it is evident that $J_{\mathrm{SC}}$ increases gradually with increasing diameter except for a local maximum at a diameter of $140 \mathrm{~nm} .{ }^{75}$ The $J_{\mathrm{SC}}$ obtained from simulation agrees well with the $J_{\mathrm{SC}}$ obtained from the photocurrent measured on a single NW device (Figure 10B, red points). The overall increase in $J_{\mathrm{SC}}$ for larger NWs results from an increased number of 
absorption peaks due to additional resonant modes emerging at longer wavelengths (Figure 10A). Smaller NW cavities support larger optical antenna effects, and this effect accounts for the local maximum in $J_{\mathrm{SC}}$ (Figure 10B, dashed black). In addition, the photocurrent per unit volume increases steadily as the size of a NW becomes smaller and, in particular, this value increases dramatically for devices with diameters less than $200 \mathrm{~nm}$ (Figure 10B, dashed red). The noticeable increase in the total photocurrent per unit volume is due to an increasingly larger ratio of absorption cross-section to physical cross-section. These results illustrate that NW structures can efficiently localize light in nanoscale volumes. Indeed, it is possible that optical concentration effects within NW photonic cavities might lead to higher injection levels and concomitant increases in photovoltage, provided that undesirable levels of Auger recombination can be avoided. One can thus envision further exploiting this feature to realize photovoltaic devices that save cost through reduced use of absorber material.

In addition to size, the morphology-dependent absorption properties of NW devices have been investigated. It has been recently shown that NWs with rectangular cross-sections can be synthesized by growing the last n-type doped shell of a p/in Si NW at higher temperatures (cf. Section 4). ${ }^{89}$ Polarization-resolved EQE spectra for devices fabricated from such rectangular NWs reveal several new features. In the TE spectrum of the $260 \mathrm{~nm}$ diameter NW (Figure 11A, black straight), a peak centered at $570 \mathrm{~nm}$ shows a nearly-unity EQE amplitude, whereas bulk Si would require $\sim 3.3 \mu \mathrm{m}$ of material to produce the same EQE value at this wavelength. Furthermore, by comparing the EQE spectrum of a rectangular NW to the spectrum of a hexagonal NW with an equivalent size, the distinct absorption properties of the rectangular NW become apparent. While the peaks centered at $565 \mathrm{~nm}$ (TE) and $680 \mathrm{~nm}$ (TM) are observed at similar wavelengths in both hexagonal and rectangular NWs (Figure 11B, *), the peaks in the 
rectangular NW have much larger amplitudes. To understand the origin of these higher amplitude peaks their accompanying absorption mode profiles were investigated. Unlike a normal peak in the rectangular NW (Figure 11A, profile 1), the higher amplitude peak exhibits a highly symmetric mode profile (Figure 11A, profile 2). Since the latter mode matches with the cross-sectional morphology of the rectangular NW it experiences lower optical loss. ${ }^{90}$ In conclusion, design of cross-sectional morphology can provide a feasible method to enhance absorption efficiency at specific wavelengths.

Recently, the morphology-dependent absorption properties of NWs with various crosssections (i.e. square, circular, hexagonal, and triangular) were explored by performing full-field electromagnetic simulations, as shown in Figure $11 \mathrm{C}-\mathrm{E} .^{75}$ In this simulation, amorphous Si NWs were considered. The results uncovered several trends that depend on the cross-section of a NW, including a wavelength shift of peaks between 650 - $800 \mathrm{~nm}$ and a variation of $J_{\mathrm{SC}}$. However, this simulation does not reproduce the pronounced peaks at longer wavelengths for a NW with rectangular cross-section as discussed in the preceding paragraph. The discrepancy between these two reported results lies in the absorber material: crystalline Si is inherently less absorptive than amorphous Si. For more absorptive materials, the short attenuation depth of photons reduces optical feedback within the cavity, leading to a weak dependence on morphological differences. In this case, differences in absorption are due to differences in cross-sectional area of the simulated structures rather than their morphology. In general, designs for efficient NW photovoltaics could include a combination of highly absorptive and highly tunable NW optical components, where the latter could be achieved with synthetic control of morphology.

From these studies, it is clear that NWs absorb light differently from thick semiconductor films that are traditionally used for photovoltaics. The NWs subwavelength size and high- 
refractive index allow for these structures to absorb light from outside their projected area and sustain optical resonant modes with distinct spatial mode profiles. Taken together, light absorption in NWs is determined by the following: (i) absorption coefficient of the NW material, (ii) spatial profile of NW cavity modes, (iii) optical antenna effect, and (iv) wavelength matching between NW cavity modes and irradiance of the solar spectrum. We see considerable promise in further tuning absorption in NWs through synthetic manipulations directed at controlling not only the cross-sectional but also the internal morphology of NWs.

\section{Assembly and Scaling of Nanowires for Photovoltaic Applications}

Single NW photovoltaics are an ideal platform for elucidating fundamental factors dictating performance ${ }^{34}$ and can be used to power nanoelectronic elements. ${ }^{36}$ Nevertheless, one must consider the potential for assembly and scaling of these functional elements into arrays that will be required for general purpose solar cells. As outlined at the beginning of this review, we have proposed a 'multi-junction' solar cell consisting of stacked NWs (Figure 1), each of which has been synthetically optimized to absorb a significant fraction of the solar spectrum.

Significantly, recent experimental results have begun to address the prospects for integration and assembly of large numbers of NWs into efficient photovoltaic arrays. ${ }^{60,61,78}$ As an initial step, core/multi-shell NW elements were integrated in parallel and it was shown that this multi-NW device (Figure 12A) retains good 'cell' characteristics (e.g., $V_{\mathrm{OC}}$ and FF are nearly constant) with increasing number of NW elements. Indeed, the FF for a device consisting of 8 NWs, $69.3 \%$, is similar to the best value attained on single NW elements, $72.0 \%$. Central to the potential for scaling, an increase in $I_{\mathrm{SC}}$ from 191 to $1723 \mathrm{pA}$ was observed as the number of NWs 
in a device was increased from 1 to 8 , respectively (Figure 12A). Critically, this increase in absolute $I_{\mathrm{SC}}$ was accompanied by preservation of $J_{\mathrm{SC}}$ to within $3 \%$ of that for the best single NW device. Furthermore, recent FDTD simulation results across a range of NW diameters show that the current density of a single-layer array of these close-packed NW building blocks is within $15 \%$ of that of a single NW (Figure 12B). Interestingly, this correspondence holds even for NW arrays with up to $30 \%$ void space, ${ }^{34}$ meaning that efficient NW solar cells could be tolerant of incomplete filling fraction. Moreover, a recent work simulated the absorption properties of a-Si NW arrays ${ }^{78}$ and, in particular, determined that absorption $\left(J_{\mathrm{SC}}\right)$ can be enhanced by appropriate choice of pitch size (Figure 12C). Together, these studies show that there is considerable promise in exploring light coupling and propagation within NW arrays. One significant advantage of an assembled NW solar cell is that NW size, morphology, and material composition can be tuned in addition to and independent of the array geometry/spacing, thus offering several strategies for control of light absorption in these systems. Development of larger arrays should be able to exploit reported advances in assembly of dense parallel nanowires on the micrometer to several inch scale, where the nanowires have also exhibited good electronic properties. ${ }^{60,61}$

For purposes of enhancing current density and thereby efficiency, we propose a new paradigm which involves layering and interconnecting optimized core/multi-shell NW components. Demonstrating the potential of this new concept, recent work has shown how a well-aligned vertical stack of 2 p/pin core-shell NWs can be assembled on a quartz substrate and connected electrically in parallel (Figure 12D, inset). Transport measurements for such a device yield a $J_{\mathrm{SC}}$ of $14.0 \mathrm{~mA} / \mathrm{cm}^{2}$, a $1.4 \mathrm{x}$ increase compared to the best single NW device. Significantly, the experimental EQE spectrum for this double NW device (Figure 12D) shows that peaks coincide in wavelength with those for a single NW device and that EQE amplitudes 
for the former are increased by a factor of $1.0-2.0$ across the spectrum. Notably, FDTD simulations reproduce the EQE enhancement for the double NW stack configuration and predict a comparable $41 \%$ increase in $J_{\mathrm{SC}}$. Finally, additional simulations have shown that a layered stack of five NWs can yield $J_{\mathrm{SC}}$ values of 24 and $>40 \mathrm{~mA} / \mathrm{cm}^{2}$ without and with a BSR, respectively (Figure 12E). These values coupled with the high $V_{\mathrm{OC}}$ values reported for the single core/multi-shell NW devices (Section 5), which have been shown to be preserved upon parallel integration (Figure 12A), could yield power-conversion efficiencies $>15 \%$.

\section{Conclusions and Prospects for Future Research}

Future studies must not only explore synthesis of new nanoscale materials ${ }^{34,59,94}$ but also develop robust methods for assembly of large area arrays for photovoltaic applications. Challenges and opportunities exist for the development of assembled NW photovoltaic arrays. For example, while existing transfer techniques ${ }^{60,61,95}$ can pattern NWs over large-areas and at high density, more desirable methods should provide control over array pitch regardless of NW size or morphology. Furthermore, to reduce packing defects during assembly of NW arrays, methods should maximize end-to-end registry of NWs and reject small NW fragments leading to voids. In addition, NW design ${ }^{96}$ and synthesis efforts should minimize NW heterogeneity with regards to size and doping, because variations in these parameters can limit the power conversion efficiency of the system ${ }^{97}$. Finally, although the total systems cost of an assembled NW photovoltaic is difficult to quantify rigorously, synthetic advances including nanowire growth by aerotaxy $^{98}$, use of cheaper metal catalysts and lower temperature processes ${ }^{52}$ will likely be a primary driver of cost reductions. Ultimately, the assembly and large-scale integration of diverse 
NW building blocks could become a viable strategy for development of efficient and costeffective solar cells. 


\section{References}

1. International Energy Outlook 2011, U. S. Energy Information Administration, 2011.

2. Renewables 2011, Global Status Report, Renewable Energy Policy Network, 2011.

3. D. M. Powell, M. T. Winkler, H. J. Choi, C. B. Simmons, D. B. Needleman, and T. Buonassisi, Energy Environ. Sci., 2012, 5, 5874-5883.

4. N. S. Lewis, Science, 2007, 315, 798-801.

5. N. S. Lewis and G. Crabtree, Basic Research Needs for Solar Energy Utilization, U. S. Department of Energy Office of Science, 2005.

6. Form EIA-63B, "Annual Photovoltaic Module/Cell Manufacturers Survey," U.S. Energy Information Administration, 2006.

7. D. Ginley, M. A. Green and R. Collins, MRS Bull., 2008, 33, 355-364.

8. M. A. Green, Third Generation Photovoltaics: Advanced Solar Energy Conversion, Springer Science, Boston, 2003.

9. Next Generation Photovoltaics: High efficiency through full spectrum utilization, ed. A. Marti and A. Luque, Institute of Physics Publishing, Bristol, 2003.

10. P. V. Kamat, J. Phys. Chem. C, 2008, 112, 18737-18753.

11. D. L. Klein, R. Roth, A. K. L. Lim, A. P. Alivisatos and P. L. McEuen, Nature, 1997, 389, 699-701.

12. S. Nie and R. N. Zare, Ann. Rev. Biophys Biomolecular Structure, 1997, 26, 567-596.

13. D. V. Talapin, J. S. Lee, M. V. Kovalenko and E. V. Shevchenko, Chem. Rev., 2010, 110, 389-458.

14. T. W. Odom, J. Huang, P. Kim and C. M. Lieber, Nature, 1998, 391, 62-64. 
15. H. W. Zhu, J. Q. Wei, K. L. Wang and D. H. Wu, Sol. Energy Mater. Sol. Cells, 2009, 93, 1461-1470.

16. L. B. Hu, D. S. Hecht and G. Gruner, Chem. Rev., 2010, 110, 5790-5844.

17. M. S. Fuhrer, J. Nygard, L. Shih, M. Forero, Y. G. Yoon, M. S. C. Mazzoni, H. J. Choi, J. Ihm, S. G. Louie, A. Zettl and P. L. McEuen, Science, 2000, 288, 494-497.

18. M. S. Dresselhaus, G. Dresselhaus, R. Saito and A. Jorio, Phys. Reports - Review Section of Physics Letters, 2005, 409, 47-99.

19. A. H. Castro Neto, F. Guinea, N. M. R. Peres, K. S. Novoselov and A. K. Geim, Rev. Mod. Phys., 2009, 81, 109-162.

20. A. K. Geim, Science, 2009, 324, 1530-1534.

21. C. M. Lieber, Solid State Comm., 1998, 107, 607-616.

22. J. Hu, T. W. Odom and C. M. Lieber, Acc. of Chem. Res., 1999, 32, 435-445.

23. X. Duan, Y. Huang, Y. Cui, J. Wang and C. M. Lieber, Nature, 2001, 409, 66-69.

24. B. Tian, T. J. Kempa and C. M. Lieber, Chem. Soc. Rev., 2009, 38, 16-24.

25. W. Lu and C. M. Lieber, J. Appl. Phys. D, 2006, 39, 387-406.

26. Y. Li, F. Qian, J. Xiang and C. M. Lieber, Mater. Today, 2006, 9, 18-27.

27. L. Lauhon, M. Gudiksen, D. Wang and C. M. Lieber, Nature, 2002, 420, 57-61.

28. M. A. Green, Prog. Photovoltaics: Research and Applications, 2001, 9, 123-135.

29. C. Brabec, MRS Bull., 2005, 30, 50-52.

30. G. Conibeer, Mater. Today, 2007, 10, 42-50.

31. A. Polman and H. A. Atwater, Nat. Mater., 2012, 11, 174-177.

32. W. Shockley and H. J. Queisser, J. Appl. Phys., 1961, 32, 510-519. 
33. H. Cotal, C. Fetzer, J. Boisvert, G. Kinsey, R. King, P. Hebert, H. Yoon and N. Karam, Energ. Environ. Sci., 2009, 2, 174-192.

34. T. J. Kempa, J. F. Cahoon, S.-K. Kim, R. W. Day, D. C. Bell, H.-G. Park and C. M. Lieber, Proc. Natl. Acad. Sci. USA, 2012, 109, 1407-1412.

35. T. J. Kempa, B. Tian, D. Kim, J. Hu, X. Zheng and C. M. Lieber, Nano Lett., 2008, 8, 3456-3460.

36. B. Tian, X. Zheng, T. J. Kempa, Y. Fang, N. Yu, G. Yu, J. Huang and C. M. Lieber, Nature, 2007, 449, 885-890.

37. B. Tian, T. Cohen-Karni, Q. Qing, X. Duan, P. Xie and C. M. Lieber, Science, 2010, 329, 831-834.

38. T. Cohen-Karni, B. P. Timko, L. E. Weiss and C. M. Lieber, Proc. Natl. Acad. Sci. USA, 2009, 106, 7309-7313.

39. W. Lu, J. Xiang, B. P. Timko, Y. Wu and C. M. Lieber, Proc. Natl. Acad. Sci. USA, 2005, 102, 10046-10051.

40. F. Qian, S. Gradecak, Y. Li, C. Y. Wen and C. M. Lieber, Nano Lett., 2005, 5, 2287-2291.

41. C. M. Lieber, MRS Bull., 2003, 28, 486-491.

42. J. D. Meindl, Q. Chen and J. A. Davis, Science, 2001, 293, 2044-2049.

43. J. R. Heath, P. J. Kuekes, G. S. Snider and R. S. Williams, Science, 1998, 280, 1716-1721.

44. S. W. Boettcher, J. M. Spurgeon, M. C. Putnam, E. L. Warren, D. B. Turner-Evans, M. D. Kelzenberg, J. R. Maiolo, H. A. Atwater and N. S. Lewis, Science, 2010, 327, 185-187.

45. M. D. Kelzenberg, S. W. Boettcher, J. A. Petykiewicz, D. B. Turner-Evans, M. C. Putnam, E. L. Warren, J. M. Spurgeon, R. M. Briggs, N. S. Lewis and H. A. Atwater, Nat. Mater., 2010, 9, 239-244. 
46. B. M. Kayes, H. A. Atwater and N. S. Lewis, J. Appl. Phys., 2005, 97, 114302-114302-11.

47. M. Law, L. E. Greene, J. C. Johnson, R. Saykally and P. Yang, Nature Mater., 2005, 4, 455-459.

48. S. M. Sze and K. K. Ng, Physics of Semiconductor Devices, John Wiley \& Sons, Inc., Hoboken, NJ, 2007.

49. A. M. Morales and C. M. Lieber, Science, 1998, 279, 208-211.

50. J. L. Liu, S. J. Cai, G. L. Jin, S. G. Thomas and K. L. Wang, J. Crystal Growth, 1999, 200, 106-111.

51. K. Haraguchi, T. Katsuyama, K. Hiruma and K. Ogawa, Appl. Phys. Lett., 1992, 60, 745747.

52. J. Tang, Z. Huo, S. Brittman, H. Gao and P. Yang, Nat. Nanotechnol., 2011, 6, 568-572.

53. P. Caroff, K. A. Dick, J. Johansson, M. E. Messing, K. Deppert and L. Samuelson, Nat. Nanotechnol., 2009, 4, 50-55.

54. F. Qian, Y. Li, S. Gradecak, H.-G. Park, Y. Dong, Y. Ding, Z. L. Wang and C. M. Lieber, Nature Mater., 2008, 7, 701-706.

55. Y. Dong, B. Tian, T. J. Kempa and C. M. Lieber, Nano Lett., 2009, 9, 2183-2187.

56. Y. Li, J. Xiang, F. Qian, S. Gradecak, Y. Wu, H. Yan, D. A. Blom and C. M. Lieber, Nano Lett., 2006, 6, 1468-1473.

57. F. Dimroth and S. Kurtz, MRS Bull., 2007, 32, 230-235.

58. B. Tian, P. Xie, T. J. Kempa, D. C. Bell and C. M. Lieber, Nat. Nanotechnol., 2009, 4, 824829.

59. S. K. Kim, R. W. Day, J. F. Cahoon, T. J. Kempa, K. Deok, H.-G. Park and C. M. Lieber, Nano Lett., 2012, 12, 4971-4976. 
60. Z. Y. Fan, J. C. Ho, Z. A. Jacobson, R. Yerushalmi, R. L. Alley, H. Razavi and A. Javey, Nano Lett., 2008, 8, 20-25.

61. A. Javey, S. W. Nam, R. S. Friedman, H. Yan, C. M. Lieber, Nano Lett., 2007, 7, 773-777.

62. M. D. Kelzenberg, D. B. Turner-Evans, M. C. Putnam, S. W. Boettcher, R. M. Briggs, J. Y. Baek, N. S. Lewis and H. A. Atwater, Energy Environ. Sci., 2011, 4, 866-871.

63. L. G. Jeffery, Handbook of Photovoltaic Science and Engineering, ed. S. Hegedus, A. Luque, Wiley, New York, 2004.

64. P. Würfel, Physics of Solar Cells, From Principles to New Concepts, Wiley-VCH Verlag GmbH \& Co. KGaA, Weinheim, 2005.

65. Y. Ke, X. Weng, J. M. Redwing, C. M. Eichfeld, T. R. Swisher, S. E. Mohney and Y. M. Habib, Nano Lett., 2009, 9, 4494-4499.

66. V. Schmidt, J. V. Wittemann and U. Gosele, Chem. Rev., 2010, 110, 361-388.

67. Y. Dan, K. Seo, K. Takei, J. H. Meza, A. Javey and K. B. Crozier, Nano Lett., 2011, 11, 2527-2532.

68. A. Goetzberger, J. Luther and G. Willeke, Solar Energ. Mat. Solar Cells, 2002, 74, 1-11.

69. O. Klutha, B. Recha, L. Houbena, S. Wiedera, G. Schöpea, C. Benekinga, H. Wagnera, A. Löfflb and H.W. Schockb, Thin Solid Films, 1999, 351, 247-253.

70. S. Koynov, M. S. Brandt and M. Stutzmann, Appl. Phys. Lett., 2006, 88, 203107-203107-3.

71. P. Bermel, C. Luo, L. Zeng, L. C. Kimerling and J. D. Joannopoulos, Optics Express, 2007, 15, 16986-17000.

72. J. Zhao and M. A. Green, IEEE Trans. Electron Devices, 1991, 38, 1925-1934.

73. S. Chhajed, M. F. Schubert, J. K. Kim and E. F. Schubert, Appl. Phys. Lett., 2008, 93, 251108-251108-3. 
74. R. Corkish, Solar Progress, 1997, 18, 16-17.

75. L. Cao, P. Fan, A. P. Vasudev, J. S. White, Z. Yu, W. Cai, J. A. Schuller, S. Fan and M. L. Brongersma, Nano Lett., 2010, 10, 439-445.

76. L. Cao, J.-S. Park, P. Fan, B. Clemens and M. L. Brongersma, Nano Lett., 2010, 10, 12291233.

77. E. S. Barnard, R. A. Pala and M. L. Brongersma, Nat. Nanotechnol., 2011, 6, 588-593.

78. D. Tham and J. R. Heath, Nano Lett., 2010, 10, 4429-4434.

79. J. Zhu, C. M. Hsu, Z. Yu, S. Fan and Y. Cui, Nano Lett., 2010, 10, 1979-1984.

80. G. Bronstrup, N. Jahr, C. Leiterer, A. Csaki, W. Fritzsche and S. Christiansen, ACS Nano., 2010, 4, 7113-7122.

81. W. F. Liu, J. I. Oh and W. Z. Shen, Nanotechnol., 2011, 22, 125705-125708.

82. K. Seo, M. Wober, P. Steinvurzel, E. Schonbrunt, Y. Dan, T. Ellenbogen and K. B. Crozier, Nano Lett., 2011, 11, 1851-1856.

83. L. Novotny and N. van Hulst, Nat. Photon., 2011, 5, 83-90.

84. A. Kinkhabwala, Z. Yu, S. Fan, Y. Avlasevich, K. Müllen and W. E. Moerner, Nature Photon., 2009, 3, 654-657.

85. M. D. Kelzenberg, D. B. Turner-Evans, B. M. Kayes, M. A. Filler, M. C. Putnam, N. S. Lewis and H. A. Atwater, Nano Lett., 2008, 8, 710-714.

86. P. L. Gourley, Nature, 1994, 371, 571-577.

87. J.-H. Kang, M.-K. Seo, S-K. Kim, S.-H. Kim, M.-K. Kim, H.-G. Park, K.-S. Kim and Y.-H. Lee, Opt. Express, 2009, 17, 6074-6081.

88. S-K. Kim, S.-H. Kim, G.-H. Kim, H.-G. Park, D.-J. Shin and Y.-H. Lee, Appl. Phys. Lett., 2004, 84, 861-863. 
89. B. Cho, J. Bareño, Y. L. Foo, S. Hong, T. Spila, I. Petrov and J. E. Greene, J. Appl. Phys., 2008, 103, 123530-123530-10.

90. H.-Y. Ryu, S.-H. Kim, H.-G. Park, J.-K. Hwang and Y.-H. Lee, Appl. Phys. Lett., 2002, 80, 3883-3885.

91. L. J. Lauhon, M. S. Gudiksen and C. M. Lieber, Phil. Trans. R. Soc. Lond. A, 2004, 362, 1247-1260.

92. L. Cao, J. S. White, J.-S. Park, J. A. Schuller, B. M. Clemens and M. L. Brongersma, Nat. Mater., 2009, 8, 643-647.

93. E. C. Garnett, M. L. Brongersma, Y. Cui and M. D. McGehee, Ann. Rev. Mater. Res., 2011, 41, 269-295.

94. M. J. Bierman and S. Jin, Energy Environ. Sci., 2009, 2, 1050-1059.

95. G. Yu, A. Cao and C. M. Lieber, Nat. Nanotechnol., 2007, 2, 372-377.

96. J. D. Christesen, X. Zhang, C. W. Pinion, T. A. Celano, C. J. Flynn and J. F. Cahoon, Nano. Lett., 2012, 12, 6024-6029.

97. J. M. Foley, M. J. Price, J. I Feldblyum and S. Maldonado, Energy Environ. Sci., 2012, 5, 5203-5220.

98. M. Heurlin, M. H. Magnusson, D. Lindgren, M. Ek, L. R. Wallenberg, K. Deppert and L. Samuelson, Nature, 2012, 492, 90-94. 
B

A
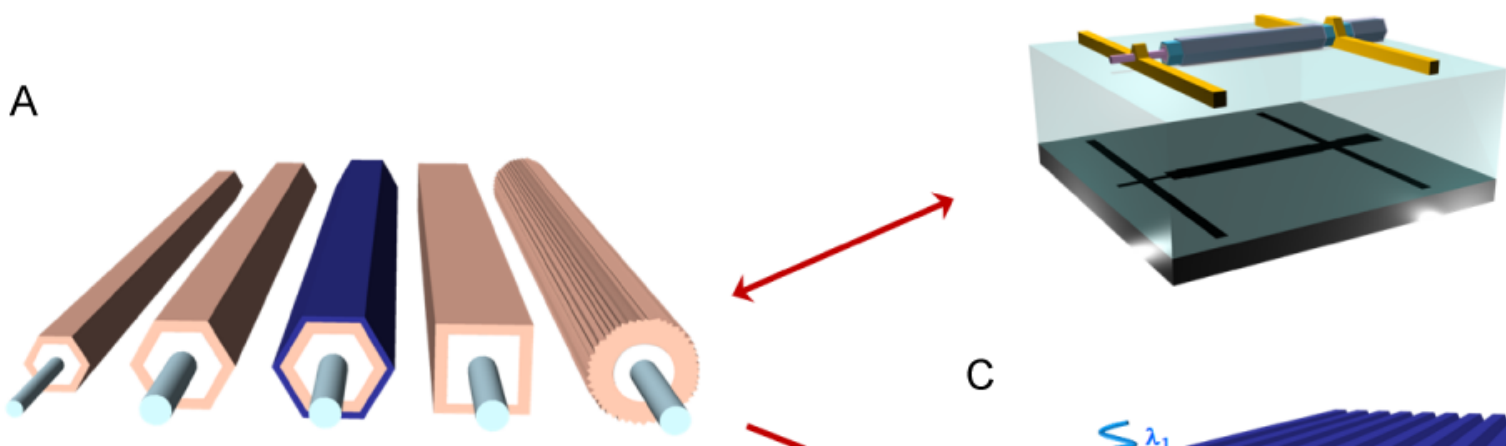

C

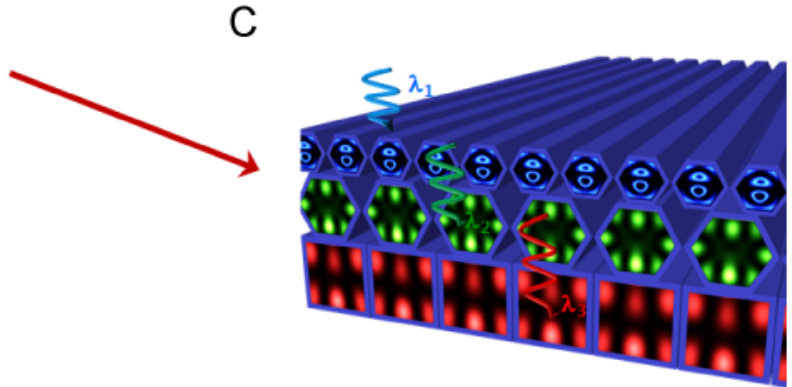

Fig. 1 Bottom-up design of NW based solar cells. (A) Schematic of coaxial NW building blocks (blue indicates p-type doped core and beige indicates n-type doped shells). (B) Schematic of typical silicon core/shell NW device fabricated from one NW building block from (A) on arbitrary substrate with integrated back-side reflector. (C) Schematic illustrating potential for new device architecture using distinct NW building blocks within each layer. Colors indicate peak wavelength of light absorbed for particular NW morphology. 


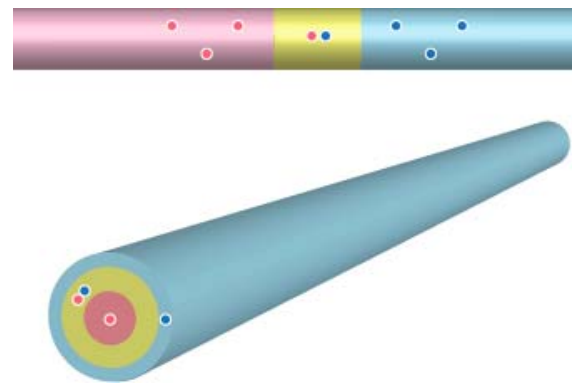

Fig. 2 Axial and radial structures form basis of NW PV. Top: Coaxial NW with carrier separation occurring in the radial direction. Bottom: Axial NW with carrier separation occurring along the axial direction. Pink indicates p-type doping, yellow indicates intrinsic (undoped), and blue indicates n-type doping. 
A

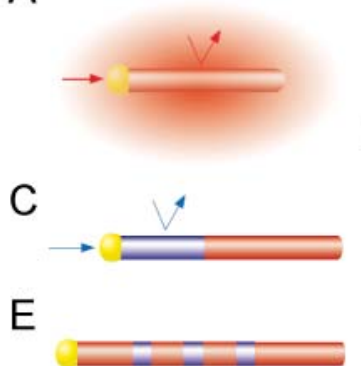

\section{B}

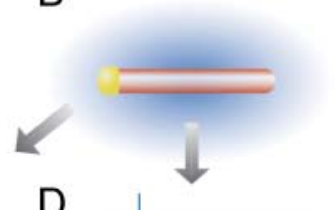

$\mathrm{D}$

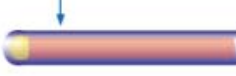

$\mathrm{F}$

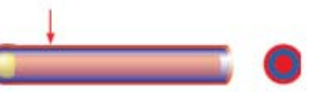

G

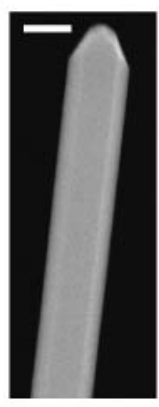

$\mathrm{H}$

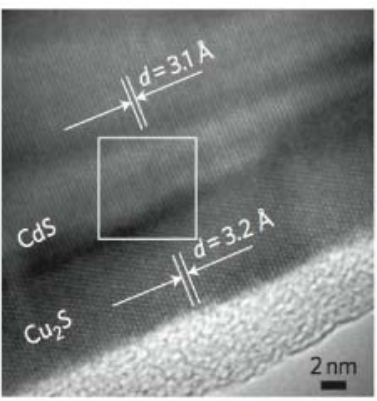

I

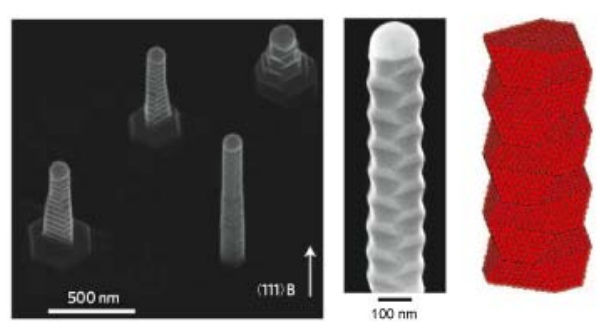

Fig. 3 Introduction to synthesis examples of techniques. (A-F) Schematics for VLS core growth (A, C, E) using metal catalyst at lower temperature, and shell growth (B, D, F) at higher temperatures. (G) SEM image of facetted Si core/shell p/in NW synthesized by CVD; scale bar $150 \mathrm{~nm}$. (H) TEM image of solution-processed core/shell $\mathrm{CdS} / \mathrm{Cu}_{2} \mathrm{~S} \mathrm{NW}$. (I) SEM images of InAs NWs exhibiting periodic coherent twin superlattice structures and their associated threedimensional atomic model. Panels A-F reproduced with permission from ref. 89 (C) The Royal Society. Panels $\mathrm{H}$ and I reproduced with permission from refs. 52 and 53 (C) Macmillan Publishers Limited. 
A

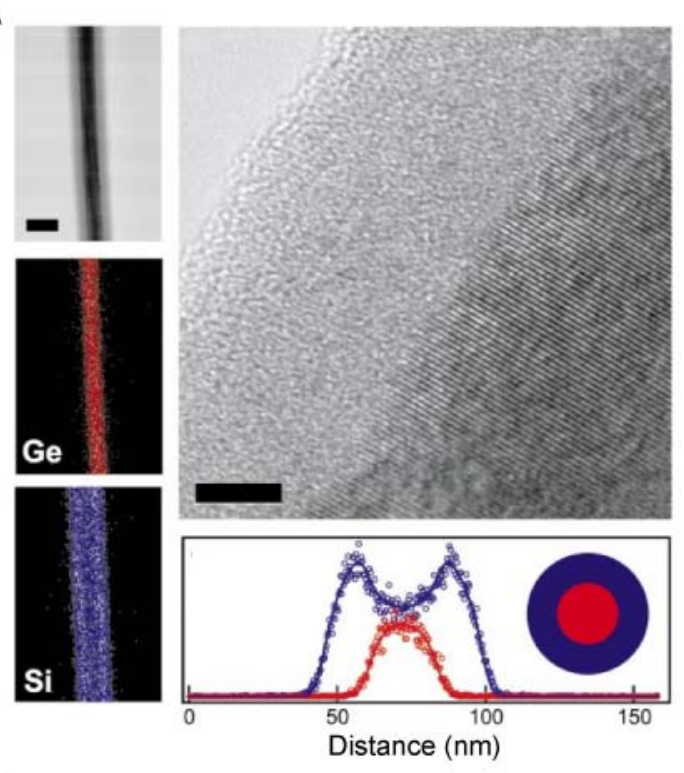

D

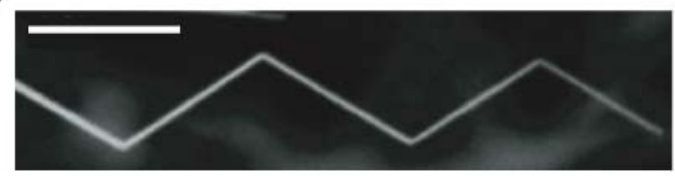

B

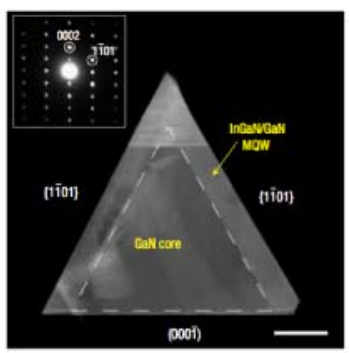

C

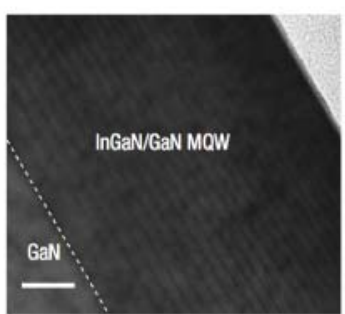

Fig. 4 Material and structural diversity. (A) Bright-field TEM images of Ge/Si core/shell NW with EDS mapping of Ge (red) and Si (blue) elements. (B) Dark-field TEM image of the cross section of an InGaN/GaN multiple quantum well NW; scale bar, $100 \mathrm{~nm}$. (C) Bright-field TEM image of an InGaN/GaN structure illustrating heterointerfaces; scale bar $10 \mathrm{~nm}$. (D) SEM image of a multiply kinked CdS NW grown by VLS mechanism; scale bar, $1 \mu \mathrm{m}$. Panels A-D reproduced with permission from refs. 27, 54, and 56 (C) Macmillan Publishers Limited. 


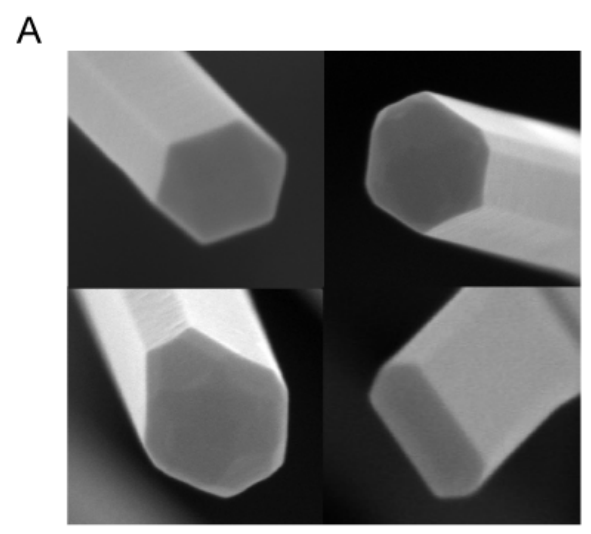

B

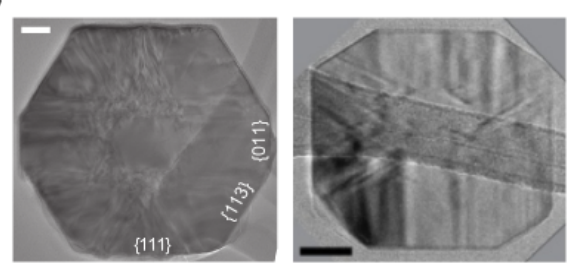

C

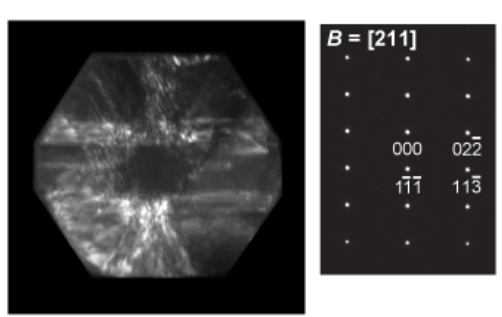

Fig. 5 Si NW PV: Characterization and control over morphology. (A) SEM images of various core/shell NW building blocks enabled by rational control of synthesis. (B) Bright-field TEM images of cross-sections of hexagonal and rectangular core/shell NWs; scale bars, $50 \mathrm{~nm}$. (C) Dark-field image of the cross-section of a core/multi-shell Si NW showing distinct crystallographic interfaces and its accompanying electron diffraction pattern along the [112] direction. Panel B left and panel C right reproduced with permission from ref. 34 (C) National Academy of Sciences of the United States of America. Panel B right reproduced with permission from ref. 57 (C) American Chemical Society. 
A
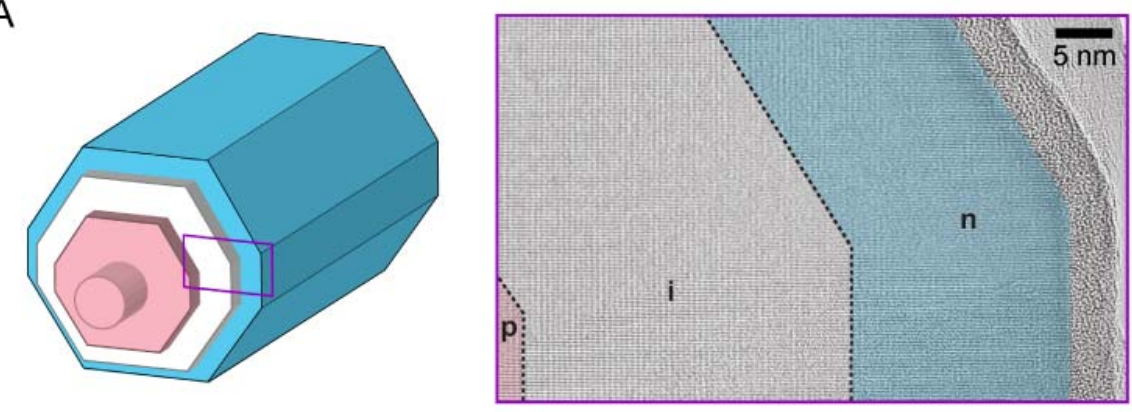

B
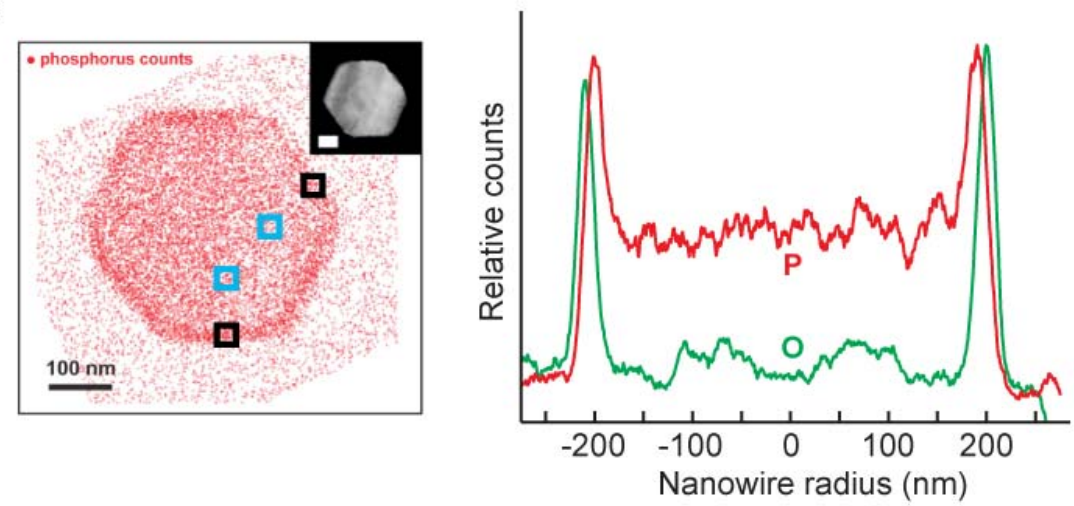

Fig. 6 Si NW PV: Control of interfaces and doping. (A) Left: Schematic of doping and morphology of p/pin core/shell NW. Right: High resolution, aberration corrected TEM image of Si shell from area indicated in purple box of schematic. Blue indicates n-type doping, white indicates intrinsic (undoped), and red indicates p-type doping. (B) EDS mapping of phosphorus atoms within Si core/shell p/pin NW acquired on STEM. Panel B reproduced with permission from ref. 34 (C) National Academy of Sciences of the United States of America. 
A

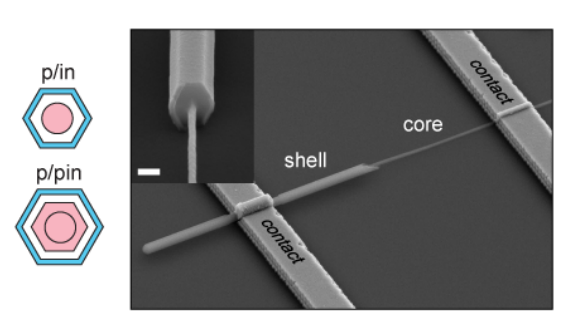

C

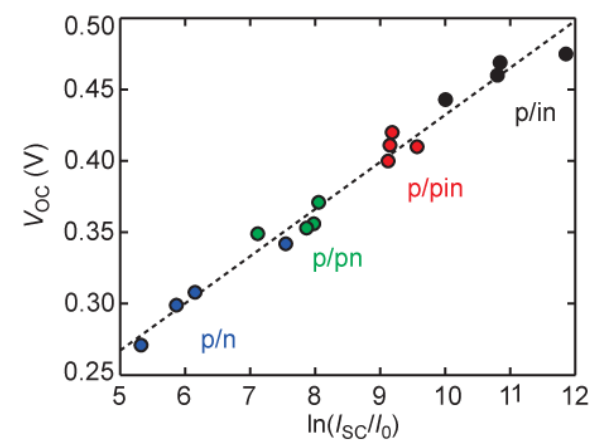

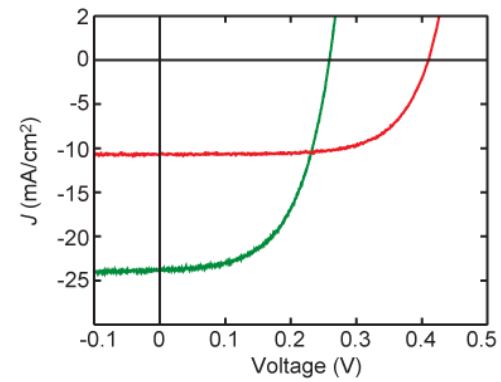

D

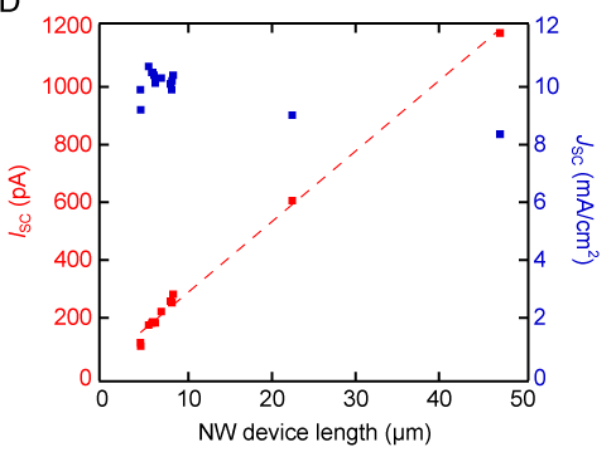

Fig. 7 Si NW PV: Fundamental performance limits and parameters. (A) Left: schematic for doping of two types of core/multi-shell NW structures; Right: representative SEM images of core/shell NW device with metallic contacts on p-type core and n-type shell; scale bar, $1 \mu \mathrm{m}$. Inset: Scale bar, $200 \mathrm{~nm}$. (B) Representative illuminated IV curves for various NW building blocks: radial NW with nanocrystalline shell, and radial NW with crystalline shell. (C) Open circuit voltage versus logarithm of ratio of short-circuit current $\left(I_{\mathrm{SC}}\right)$ to dark saturation current $\left(I_{0}\right)$ for various radial core/shell NW structures. (D) Short circuit current $\left(I_{\mathrm{SC}}\right)$ and short circuit current density $\left(J_{\mathrm{SC}}\right)$ of NWs under 1-sun illumination as a function of NW length. Panels A, C, $\mathrm{D}$, and panel B red curve reproduced with permission from ref. 34 (C) National Academy of Sciences of the United States of America. Panel B green curve reproduced with permission from ref. 36 (C) Macmillan Publishers Limited. 

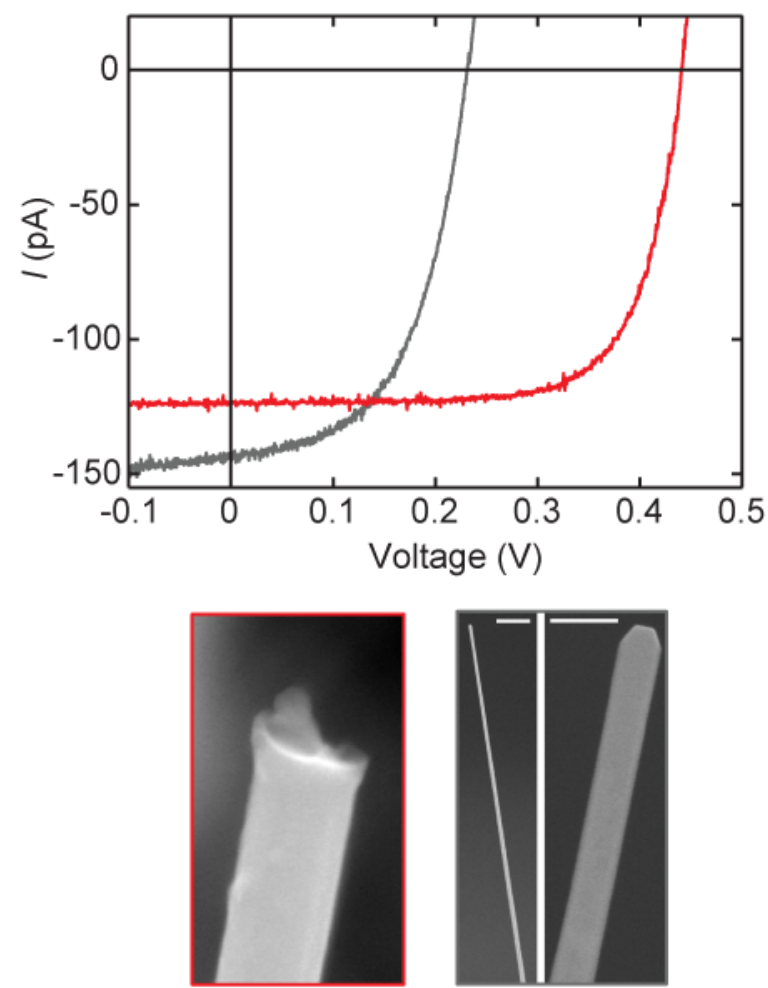

Au-catalyzed

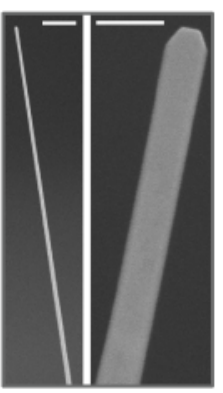

Al-catalyzed

Fig. 8 Role of metal catalyst on PV performance. Left: Illuminated IV curves for Si core/shell $\mathrm{p} / \mathrm{i} / \mathrm{n}$ devices. Red curve corresponds to device from NW where Au was etched prior to shell deposition. Grey curve corresponds to device from NW grown with an Al catalyst. Bottom: SEM images of Au-etched and Al-catalyzed NWs; scale bar, $2 \mu \mathrm{m}$ and $1 \mu \mathrm{m}$. Reproduced with permission from ref. 34 (c) National Academy of Sciences of the United States of America. 
A

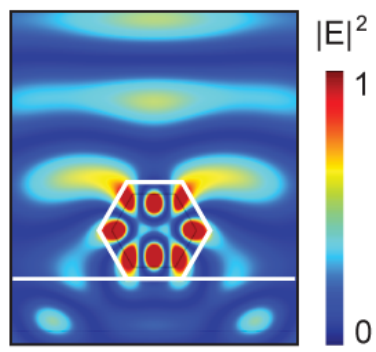

B

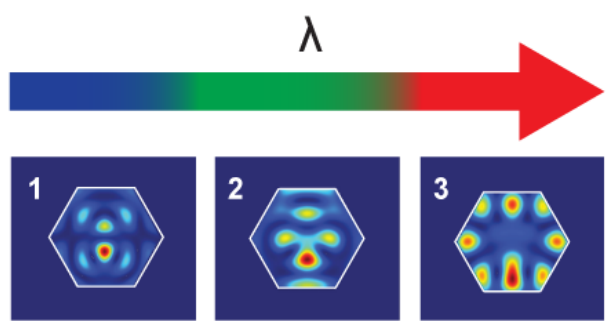

D

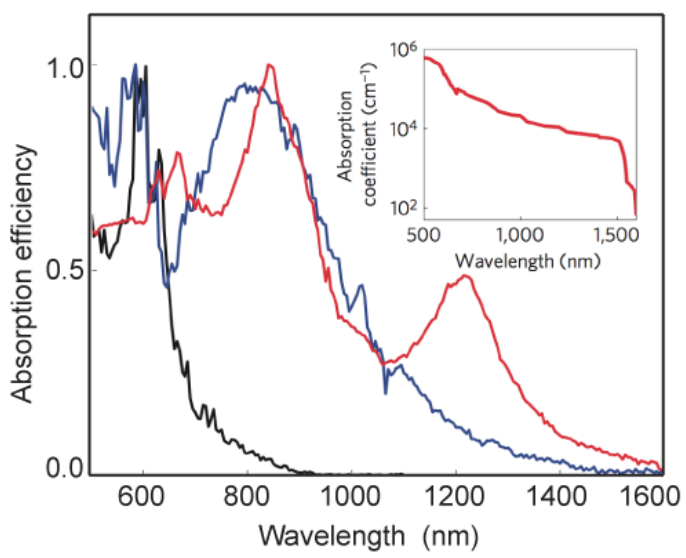

Fig. 9 Optical resonant modes in NWs and measurement of absolute EQE. (A) Electric field intensity distribution illustrating vertically incident light coupling into NW cavity. (B) Various absorption mode profiles corresponding to different peaks in EQE spectrum. Arrow indicates relative wavelength of light needed to excite resonant mode. (C) Experimental (black) and simulated (red) EQE spectrum of core/shell hexagonal NW device with height $=240 \mathrm{~nm}$. Simulated EQE spectrum of equivalent thickness of bulk Si plotted in green for comparison. (D) Relative absorption efficiency for Ge photodetector devices as function of wavelength. Panel A reproduced with permission from ref. 57 (C) American Chemical Society. Panels B and C reproduced with permission from ref. 34 (C) National Academy of Sciences of the United States of America. Panel D reproduced with permission from ref. 90 (C) Macmillan Publishers Limited. 
A

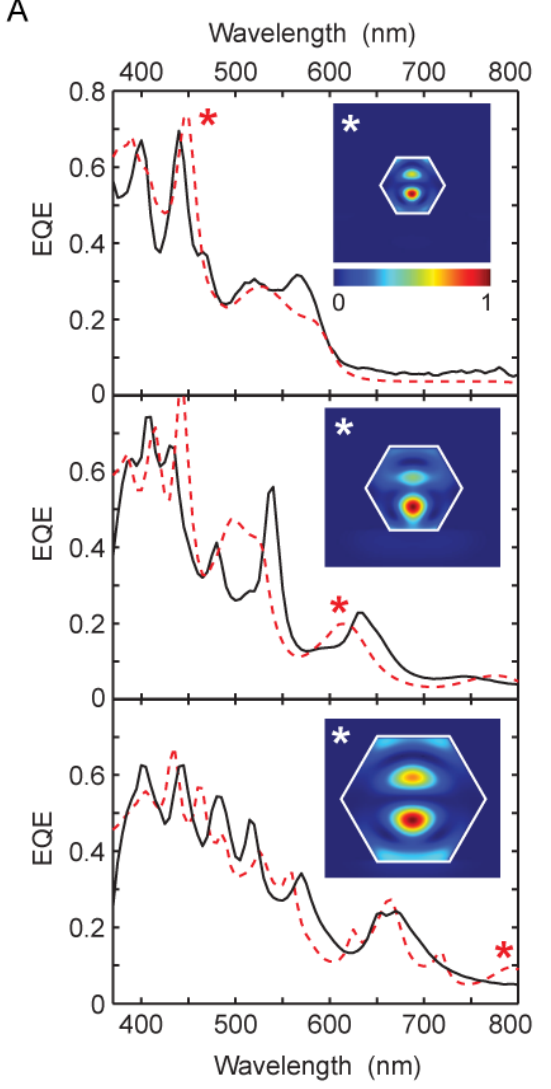

B

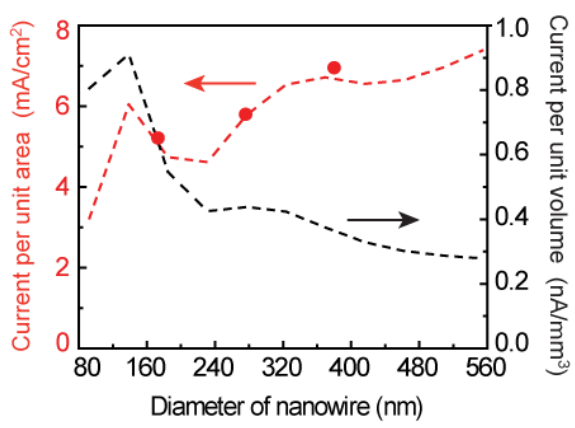

Fig. 10 Influence of size on NW spectra. (A) Experimental (black) and simulated (red) EQE spectra for three hexagonal NWs with diameters (top to bottom) of 170, 280, and $380 \mathrm{~nm}$. Inset: Fabry-Perot absorption mode corresponding to peak labeled by * in spectra. (B) Experimental values of current per unit area (red) and current per unit volume (black) for three NWs in spectra in (A). Dotted lines are simulated values for various diameters. Reproduced with permission from ref. 57 (C) American Chemical Society. 
A

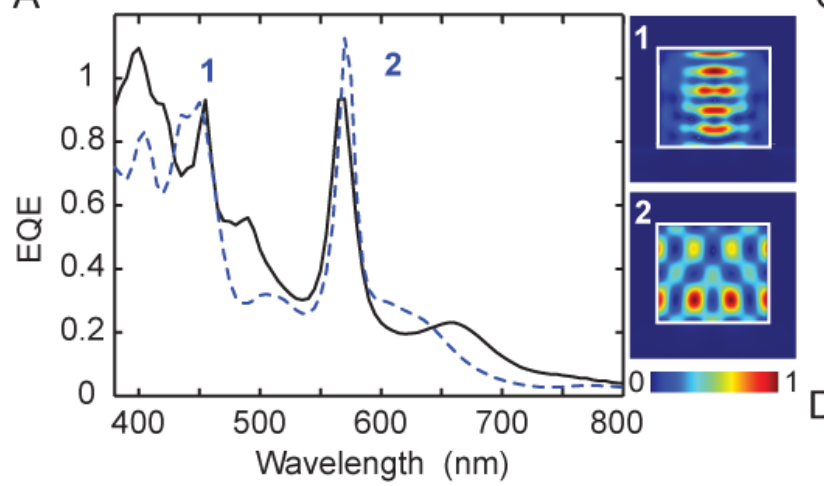

$\mathrm{B}$

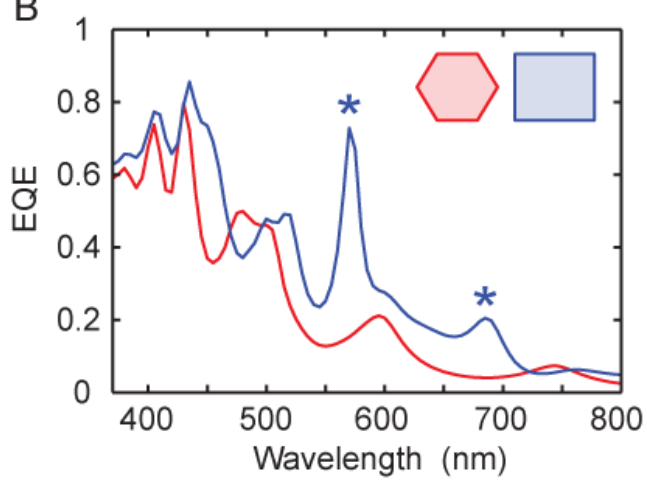

C
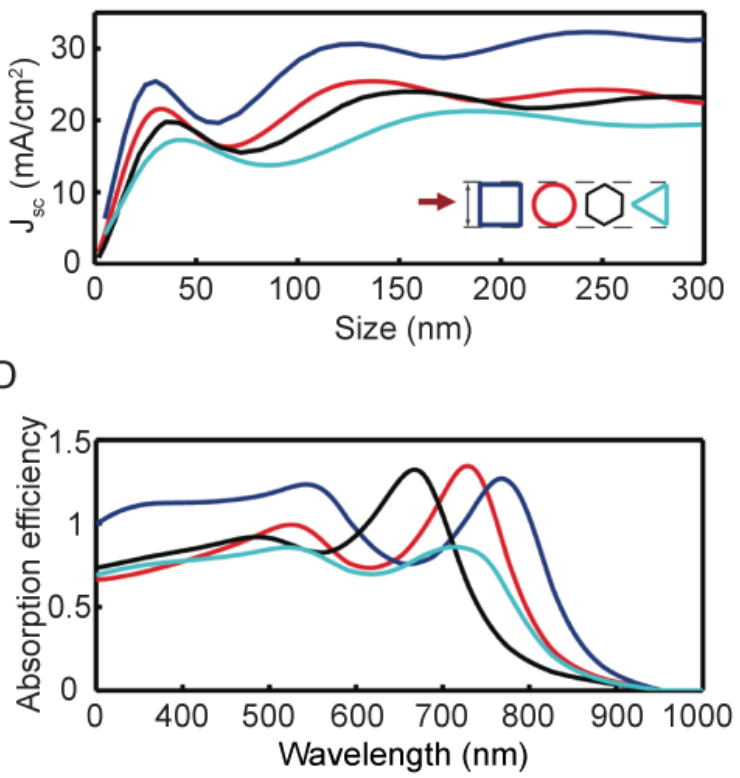

$E$

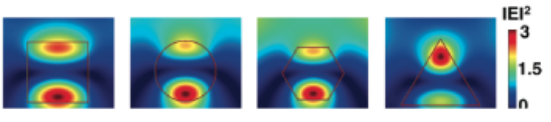

Fig. 11 Influence of nanowire morphology on absorption. (A) Tranverse-electric EQE spectrum of rectangular morphology crystalline-Si NW device. Right: Absorption mode profiles corresponding to numbered peaks in spectrum. (B) Comparison of hexagonal vs. rectangular EQE spectrum of unpolarized light. Data are from FDTD simulations which assume equivalent height and diameters for both morpholgies. (C) Simulated $J_{\mathrm{SC}}$ values as a function of NW size for various morphologies of amorphous silicon NWs. (D) Simulated absorption spectra for morphologies in (C). (E) Electric field intensity distribution for a particular resonant mode excited in the various morphologies shown in (C). Panels A and B reproduced with permission from ref. 57 (C) American Chemical Society. Panels C-E reproduced with permission from ref. 73 (C) American Chemical Society. 
A

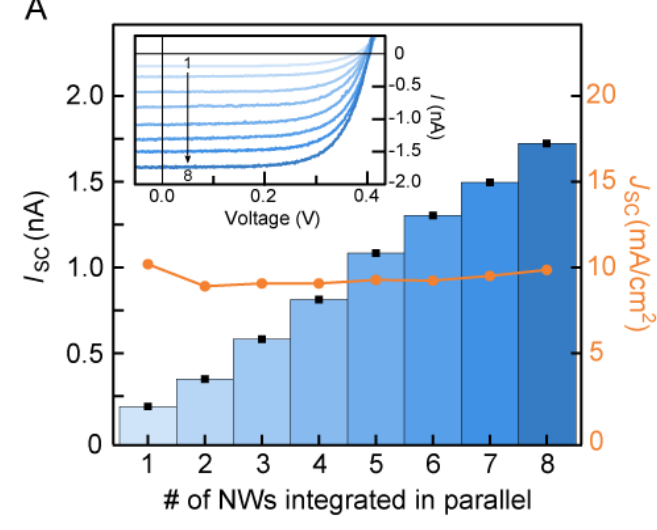

C

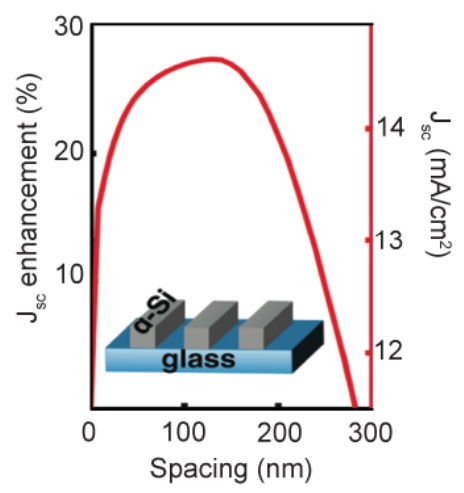

E

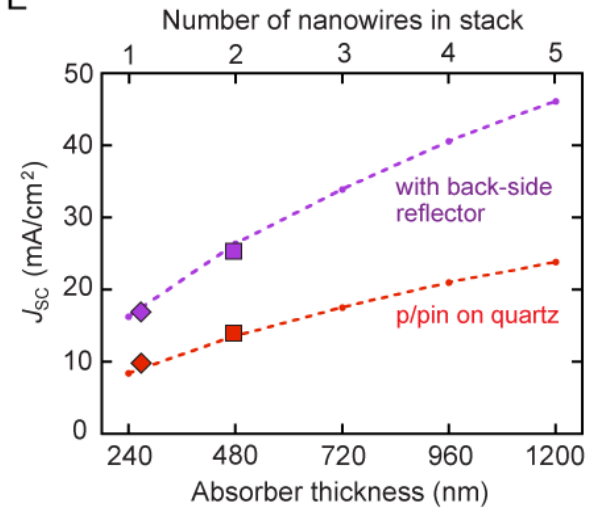

B

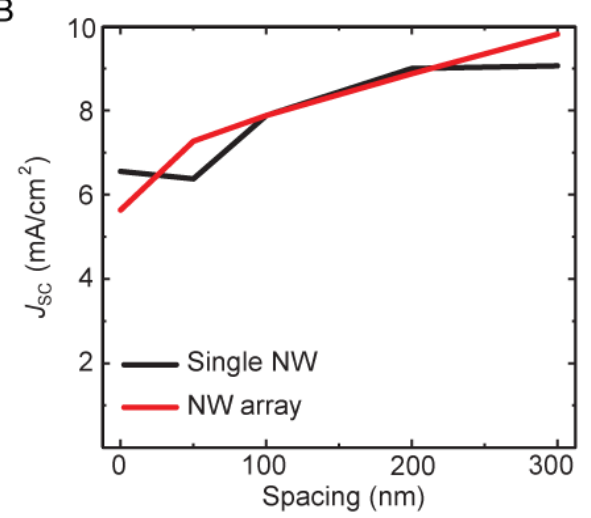

D

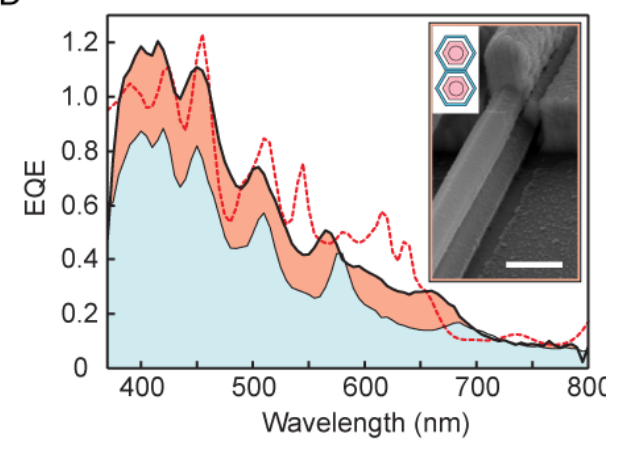

Fig. 12 Horizontal arrays and vertical stacks of NW as PV platform. (A) Photocurrent increase with increasing number of Si NWs in a parallel array. (B) Simulated $J_{\mathrm{SC}}$ values for both single NW (black) and close-packed NW array (red) as a function of NW diameter. (C) $J_{\mathrm{SC}}$ enhancement as a function of the pitch between amorphous silicon NWs in a horizontal array; absolute $J_{\mathrm{SC}}$ (red) on right. (D) Experimental (solid black) EQE spectra of single (blue) and 2 NW stack (orange); simulated EQE spectrum (dashed red line) of 2 NW stack, inset: SEM of 2 NW stack device; scale bar, $500 \mathrm{~nm}$. (E) Data and simulations of $J_{\mathrm{SC}}$ as a function of the number of NWs within a vertical stack. Panels A, D, E reproduced with permission from ref. 34 (C) National Academy of Sciences of the United States of America. Panel C reproduced with permission from ref. 76 (C) American Chemical Society. 